\title{
Real-World Pricing for a Modified Constant Elasticity of Variance Model
}

\author{
Shane M. Miller ${ }^{1}$ and Eckhard Platen ${ }^{2}$
}

June 1, 2009

\begin{abstract}
This paper considers a modified constant elasticity of variance (MCEV) model. This model uses the familiar constant elasticity of variance form for the volatility of the growth optimal portfolio (GOP) in a continuous market. It leads to a GOP that follows the power of a time-transformed squared Bessel process. This paper derives analytic real-world prices for zero-coupon bonds, instantaneous forward rates and options on the GOP that are both theoretically revealing and computationally efficient. In addition, the paper examines options on exchange prices and options on zerocoupon bonds under the MCEV model. The semi-analytic prices derived for options on zero-coupon bonds can subsequently be used to price interest rate caps and floors.
\end{abstract}

1991 Mathematics Subject Classification: primary 90A12; secondary 60G30, 62P20. JEL Classification: G10, G13

Key words and phrases: benchmark approach, real-world pricing, growth optimal portfolio, constant elasticity of variance, zero-coupon bonds, exchange prices, interest rate caps and floors.

\footnotetext{
${ }^{1}$ Citigroup Global Markets Australia Pty Ltd, 2 Park Street, Sydney NSW 2000, Australia.

${ }^{2}$ University of Technology Sydney, School of Finance \& Economics and Department of Mathematical Sciences, PO Box 123, Broadway NSW 2007, Australia.
} 


\section{Introduction}

The standard constant elasticity of variance (CEV) model has a distinguished history. It was developed in Cox \& Ross (1976), Beckers (1980), Schroder (1989), Lo, Yuen \& Hui (2000) and others, with summaries given in Cox (1996) and Shaw (1998). Empirical tests of the CEV model can be found in MacBeth \& Merville (1980), Engel \& MacBeth (1982) and Jones (2003). The CEV model has also been applied to non-standard derivatives such as lookback and barrier options in Boyle \& Tian (1999), Davydov \& Linetsky (2001), Linetsky (2004) and Lo et al. (2004). Despite this extensive research, it is only recently that discussions on the difficulties of applying risk-neutral pricing to the CEV model have appeared, including Sin (1998), Lewis (2000), Delbaen \& Shirakawa (2002), Cox \& Hobson (2005) and Heston, Loewenstein \& Willard (2007). Another study detailing the drawbacks of the risk-neutral approach for the CEV model in a modified setting is that of Heath \& Platen (2002). Here the standard CEV model is used as a model for the growth optimal portfolio (GOP) without assuming that an equivalent riskneutral probability measure exists. The resulting model for the GOP under the real-world probability measure, referred to as the modified constant elasticity of variance (MCEV) model, is the subject of this paper. The GOP is the numeraire portfolio that results in pricing under the real-world probability measure, as per Platen \& Heath (2006). In addition, the GOP can be interpreted as a global market index.

The precise form of the MCEV model is most succinctly defined as an expression for the volatility of the GOP. In words, the volatility of the GOP equals the GOP itself raised to a power and multiplied by a scalar. This is a straightforward generalisation of the classical risk-neutral Black-Scholes-Merton model with a level-dependent local volatility function. Under this MCEV model we derive a series of new analytic formulae for standard contingent claims.

First, the real-world price of a zero-coupon bond is given in terms of two independent components, one driven by the short rate, the other driven by a discounted GOP. This second component can be expressed in terms of the central chi-square distribution and is found to be a supermartingale. A corresponding formula for the instantaneous forward rate under the MCEV model is also presented and discussed. Second, the real-world price of standard European call and put options on the GOP are obtained in terms of central and non-central chi-square distributions for the MCEV model. Thus we extend the work of Heath \& Platen (2002) where numerical solutions were utilised to price zero-coupon bonds and options on the GOP. The analytic formulae derived in this paper facilitate direct comparison between real-world and risk-neutral prices, thus highlighting failures and limitations of the risk-neutral approach, also observed in Lewis (2000) and Heston, Loewenstein \& Willard (2007). Third, analytic pricing formulae are derived for European call and put options on exchange prices in terms of the doubly

non-central beta distribution, which itself is related to a ratio of two indepen- 
dent non-central chi-square distributed random variables. Lastly, we calculate real-world prices for both call and put options on zero-coupon bonds under the MCEV model. These latter results are semi-analytic in nature, yet facilitate easy transformation to prices for interest rate caps and floors.

\section{Continuous Financial Market}

We consider a continuous multi-asset market with $d+1$ primary assets. Uncertainty in this market is modelled by $d$ independent standard Wiener processes $W^{k}=\left\{W_{t}^{k}, t \in[0, T]\right\}, k \in\{1,2, \ldots, d\}$. These are defined on a filtered probability space $\left(\Omega, \mathcal{A}_{T}, \underline{\mathcal{A}}, P\right)$ with finite time horizon $T \in(0, \infty)$ and filtration $\mathcal{A}=\left(\mathcal{A}_{t}\right)_{t \in[0, T]}$ fulfilling the usual conditions, as given in Karatzas \& Shreve (1998). $P$ is the real-world probability measure. In the following we summarise the benchmark approach as developed in Platen (2002) and Platen \& Heath (2006).

First, we assume that the $i$ th savings account $B_{t}^{i}$, associated with the short rate $r_{t}^{i}$ in the $i$ th currency denomination, satisfies the equation

$$
B_{t}^{i}=B_{0}^{i} \exp \left\{\int_{0}^{t} r_{s}^{i} d s\right\}
$$

for $t \in[0, T]$ and $i \in\{0,1, \ldots, d\}$. We set $B_{0}^{i}=1$ without loss of generality.

Second, we introduce the GOP as the portfolio that achieves the maximum expected growth rate in the long run, as outlined in Platen \& Heath (2006). The GOP has also been described as the best performing portfolio. It was studied previously in Kelly (1956), Long (1990), Karatzas \& Shreve (1998), Platen (2002) and by many other researchers. We define the GOP process $S^{i, \delta_{*}}=\left\{S_{t}^{i, \delta_{*}}, t \in[0, T]\right\}$ in the $i$ th currency denomination by the stochastic differential equation (SDE)

$$
d S_{t}^{i, \delta_{*}}=S_{t}^{i, \delta_{*}}\left[r_{t}^{i}+\left|\theta_{t}^{i}\right|^{2}\right] d t+S_{t}^{i, \delta_{*}}\left|\theta_{t}^{i}\right| d W_{t}^{i}
$$

for $t \in[0, T]$ and $i \in\{0,1, \ldots, d\}$. Here $W^{i}$ is a Wiener process and $\left|\theta^{i}\right|=$ $\left\{\left|\theta_{t}^{i}\right|, t \in[0, T]\right\}$ denotes the total market price of risk in the $i$ th currency denomination. However in this paper we interpret $\left|\theta_{t}^{i}\right|$ usually as the volatility of the GOP in the $i$ th currency denomination. Platen (2005) showed that appropriately defined well-diversified portfolios represent approximate GOPs. Indeed, all well-diversified portfolios exhibit similar behaviour. Therefore, total return stock market indices such as the MSCI Growth World Stock (MSCI) Index, can be used to approximate the GOP. This implies that market observable data, such as the volatility of the MSCI Index, can be used in conjunction with the benchmark approach of Platen \& Heath (2006) for various practical applications.

Third, we define an exchange price $X_{t}^{i, j}$ as the amount one pays in units of the $i$ th primary asset at time $t$ to obtain one unit of the $j$ th primary asset. In 
equation form, an exchange price can be defined with respect to any strictly positive portfolio. In terms of the two different denominations $S_{t}^{i, \delta_{*}}$ and $S_{t}^{j, \delta_{*}}$ of the GOP, one obtains

$$
X_{t}^{i, j}=\frac{S_{t}^{i, \delta_{*}}}{S_{t}^{j, \delta_{*}}}
$$

for $t \in[0, T]$ and $i, j \in\{0,1, \ldots, d\}$. Thus exchange prices can be defined analogously to an exchange rate between different currencies, or a share price in a given currency.

Fourth, we introduce the primary security account process $S^{i, j}=\left\{S_{t}^{i, j}, t \in[0, T]\right\}$ for $i, j \in\{0,1, \ldots, d\}$ to model the $j$ th primary asset, when measured in units of the $i$ th currency. Each primary security account represents the accumulation of all income, carrying costs plus capital gains or losses achieved whilst holding the underlying primary asset. The exchange price thus provides a link between primary security accounts and savings accounts, written as

$$
S_{t}^{i, j}=X_{t}^{i, j} B_{t}^{j}
$$

for $t \in[0, T]$ and $i, j \in\{0,1, \ldots, d\}$. In this respect, one observes that under the benchmark approach, an exchange price represents more than the exchange rate between two currencies. As an example, an exchange price can also be used to represent an ex-dividend stock price in a particular currency, whilst the corresponding cum-dividend stock price in the same currency is represented by a primary security account.

Lastly, consider the discounted GOP process $\bar{S}^{i, \delta_{*}}=\left\{\bar{S}_{t}^{i, \delta_{*}}, t \in[0, T]\right\}$ in the $i$ th currency denomination, given by

$$
\bar{S}_{t}^{i, \delta_{*}}=\frac{S_{t}^{i, \delta_{*}}}{B_{t}^{i}}
$$

satisfying the SDE

$$
d \bar{S}_{t}^{i, \delta_{*}}=\bar{S}_{t}^{i, \delta_{*}}\left|\theta_{t}^{i}\right|^{2} d t+\bar{S}_{t}^{i, \delta_{*}}\left|\theta_{t}^{i}\right| d W_{t}^{i}
$$

for $t \in[0, T]$ and $i \in\{0,1, \ldots, d\}$. Thus discounting in each currency denomination, provides a natural way to separate the corresponding short rate and the market price of risk components of the GOP in any given currency denomination.

\section{Real-World Pricing}

The concept of real-world pricing outlined in Platen \& Heath (2006) involves the selection of the GOP as the numeraire portfolio and using conditional expectation with respect to the real-world probability measure $P$. We start by 
defining a contingent claim $H_{\bar{T}}^{i}$ that matures at the stopping time $\bar{T} \in[0, T]$ for $i \in\{0,1, \ldots, d\}$ as an $\mathcal{A}_{\bar{T}}$-measureable, non-negative payoff that possesses a finite expectation when given in units of the GOP.

Corollary 2.1 [Platen (2002)] The real-world price $U_{t}^{i, H_{\bar{T}}^{i}}$ in the ith denomination is obtained at time $t$ by the formula

$$
U_{t}^{i, H_{\bar{T}}^{i}}=E\left[\frac{S_{t}^{i, \delta_{*}}}{S_{\bar{T}}^{i, \delta_{*}}} H_{\bar{T}}^{i} \mid \mathcal{A}_{t}\right]
$$

for $t \in[0, \bar{T}]$ and $i \in\{0,1, \ldots, d\}$.

For a replicable contingent claim $H_{\bar{T}}^{i}$ this is also the minimal possible price because of the supermartingale property of benchmarked non-negative portfolios, as explained in (Platen \& Heath 2006).

To understand the link to risk-neutral pricing, consider the candidate RadonNikodym derivative process $\Lambda^{i, \theta}=\left\{\Lambda_{t}^{i, \theta}, t \in[0, T]\right\}$ in the $i$ th currency denomination for the putative risk-neutral measure $P_{i, \theta}$. From Karatzas \& Shreve (1998), for the case of a complete market $\Lambda^{i, \theta}$ takes the form

$$
\Lambda_{t}^{i, \theta}=\left.\frac{d P_{i, \theta}}{d P}\right|_{\mathcal{A}_{t}}=\frac{B_{t}^{i}}{S_{t}^{i, \delta_{*}}} \frac{S_{0}^{i, \delta_{*}}}{B_{0}^{i}}=\frac{\bar{S}_{0}^{i, \delta_{*}}}{\bar{S}_{t}^{i, \delta_{*}}}
$$

for $t \in[0, \bar{T}]$ and $i \in\{0,1, \ldots, d\}$ with initial value $\Lambda_{0}^{i, \theta}=1$. In fact, the candidate Radon-Nikodym derivative is found from (2.6) and (2.8) to equal

$$
\Lambda_{t}^{i, \theta}=\exp \left\{-\frac{1}{2} \int_{0}^{t}\left|\theta_{s}^{i}\right|^{2} d s-\int_{0}^{t}\left|\theta_{s}^{i}\right| d W_{s}^{i}\right\}
$$

for $t \in[0, T]$ and $i \in\{0,1, \ldots, d\}$. Obviously, $\Lambda^{i, \theta}$ as given in $(2.9)$ is an $(\underline{\mathcal{A}}, P)$ local martingale. Whether or not (2.9) describes a martingale will depend upon the nature of the volatility of the GOP $\left|\theta_{t}^{i}\right|$. For models where the candidate risk-neutral measure $P_{i, \theta}$ and the real-world measure $P$ are equivalent and the Radon-Nikodym derivative process $\Lambda^{i, \theta}$ is an $(\underline{\mathcal{A}}, P)$-martingale, the real-world pricing formula (2.7) simplifies via Bayes' Theorem to the standard risk-neutral pricing formula of

$$
U_{t}^{i, H_{\bar{T}}^{i}}=E\left[\frac{\Lambda_{\bar{T}}^{i, \theta}}{\Lambda_{t}^{i, \theta}} \frac{B_{t}^{i}}{B_{\bar{T}}^{i}} H_{\bar{T}}^{i} \mid \mathcal{A}_{t}\right]=E_{i, \theta}\left[\frac{B_{t}^{i}}{B_{\bar{T}}^{i}} H_{\bar{T}}^{i} \mid \mathcal{A}_{t}\right]
$$

for $t \in[0, \bar{T}]$ and $i \in\{0,1, \ldots, d\}$. Here $E_{i, \theta}$ denotes conditional expectation with respect to the risk-neutral probability measure $P_{i, \theta}$. 
It should be noted that under the MCEV model discussed in this paper, the assumptions underlying the risk-neutral pricing formula are not satisfied, and thus the second equality in (2.10) does not hold. In contrast, the real-world pricing formula (2.7) remains applicable under the MCEV model. This does not mean that arbitrage exists, as defined in Platen \& Heath (2006). The definition of arbitrage used within the real-world pricing framework of Platen \& Heath (2006) is that "strictly positive profits can be generated under limited liability with strictly positive probability from zero initial wealth". This is different to the no-freelunch-with-vanishing-risk NFLVR condition of Delbaen \& Schachermayer (1994). One can argue, along with Loewenstein \& Willard (2000), that the NFLVR condition is too restrictive since the existence of an equivalent probability measure is not necessary to capture the true economic spirit of no-arbitrage. Hence, the NFLVR is mathematically convenient, but not an economic necessity.

\section{Modified CEV Dynamics}

Historically the standard CEV model was developed to model stock prices with level-dependent volatility. This paper differs from that approach in that it follows Heath \& Platen (2002) in studying a modified constant elasticity of variance (MCEV) model for the GOP. It is modified in the sense that the CEV form is used to model the volatility of the GOP rather than in the diffusion term of a stock price. The MCEV model for the GOP is obtained when its volatility takes the form

$$
\left|\theta_{t}^{i}\right|=\xi_{i}\left(S_{t}^{i, \delta_{*}}\right)^{\beta_{i}-1}
$$

for $t \in[0, T]$ with exponent $\beta_{i} \in(-\infty,+\infty)$ and a constant scaling parameter $\xi_{i}>0, i \in\{0,1, \ldots, d\}$. One observes that in this form the volatility of the GOP is stochastic when $\beta_{i} \neq 1$, since it depends on the level of the GOP itself. Combining (3.1) with (2.2) leads to an SDE for the GOP of the form

$$
d S_{t}^{i, \delta_{*}}=\left[r_{t}^{i} S_{t}^{i, \delta_{*}}+\xi_{i}^{2}\left(S_{t}^{i, \delta_{*}}\right)^{2 \beta_{i}-1}\right] d t+\xi_{i}\left(S_{t}^{i, \delta_{*}}\right)^{\beta_{i}} d W_{t}^{i}
$$

for $t \in[0, T]$ and $i \in\{0,1, \ldots, d\}$. Hence the drift of the GOP is also stochastic. Obviously, in the case when $\beta_{i}=1$ the dynamics reduce to geometric Brownian motion, as in the Black-Scholes-Merton model.

A different interpretation of the MCEV model is that of a stochastic volatility model with perfect correlation between the driving processes of the GOP and its squared volatility. To see this we set $v_{t}^{i}=\left|\theta_{t}^{i}\right|^{2}=\xi_{i}^{2}\left(S_{t}^{i, \delta_{*}}\right)^{2\left(\beta_{i}-1\right)}$ as the square of the volatility of the GOP in the $i$ th denomination. Then using (2.2), (3.1) and the Itô formula we can write the SDE for the squared volatility of the GOP as

$$
d v_{t}^{i}=\left(\beta_{i}-1\right)\left[2 r_{t}^{i} v_{t}^{i}+\left(2 \beta_{i}-1\right)\left(v_{t}^{i}\right)^{2}\right] d t+2\left(\beta_{i}-1\right)\left(v_{t}^{i}\right)^{3 / 2} d W_{t}^{i}
$$


for $t \in[0, T]$ and $i \in\{0,1, \ldots, d\}$.

Heath \& Platen (2002) show under an MCEV model that it is possible to reexpress the dynamics of the GOP as a power of an underlying square root process. In detail, they showed that

$$
S_{t}^{i, \delta_{*}}=\left(Z_{t}^{i}\right)^{\frac{1}{2\left(1-\beta_{i}\right)}}
$$

where $Z^{i}=\left\{Z_{t}^{i}, t \in[0, T]\right\}$ is a square root process satisfying the SDE

$$
d Z_{t}^{i}=\left[\xi_{i}^{2}\left(1-\beta_{i}\right)\left(3-2 \beta_{i}\right)+2\left(1-\beta_{i}\right) r_{t}^{i} Z_{t}^{i}\right] d t+2 \xi_{i}\left(1-\beta_{i}\right) \sqrt{Z_{t}^{i}} d W_{t}^{i}
$$

for $t \in[0, T]$ and $i \in\{0,1, \ldots, d\}$ with initial value $Z_{0}^{i}=\left(S_{0}^{i, \delta_{*}}\right)^{2\left(1-\beta_{i}\right)}>0$. In turn, because of the close relationship between square root and squared Bessel (BESQ) processes, the GOP can be expressed as a functional of a BESQ process. To illustrate this, we introduce the scaling function

$$
A_{t}^{i}=A_{0}^{i} \exp \left\{2\left(1-\beta_{i}\right) r_{t}^{i} t\right\}
$$

for $t \in[0, T]$ and $i \in\{0,1, \ldots, d\}$. Let us use the time change $\tau^{i}=\left\{\tau_{t}^{i}, t \in[0, T]\right\}$, defined as

$$
\tau_{t}^{i}=\tau_{0}^{i}+\int_{0}^{t} \frac{\xi_{i}^{2}\left(1-\beta_{i}\right)^{2}}{A_{s}^{i}} d s=\tau_{0}^{i}+\frac{\xi_{i}^{2}\left(1-\beta_{i}\right)}{2 r_{i} A_{0}^{i}}\left(1-\exp \left\{-2\left(1-\beta_{i}\right) r_{t}^{i} t\right\}\right)
$$

for $t \in[0, T]$ and $i \in\{0,1, \ldots, d\}$. Therefore defining

$$
X_{\tau_{t}^{i}}^{i}=\frac{Z_{t}^{i}}{A_{t}^{i}}
$$

yields in the transformed time scale of (3.7), an SDE of the form

$$
d X_{\tau_{t}^{i}}^{i}=\frac{3-2 \beta_{i}}{1-\beta_{i}} d \tau_{t}^{i}+\sqrt{4 X_{\tau_{t}^{i}}^{i}} d \hat{W}_{\tau_{t}^{i}}^{i}
$$

for $\tau_{t}^{i} \in\left[\tau_{0}^{i}, \tau_{T}^{i}\right]$ and $i \in\{0,1, \ldots, d\}$, driven by the Wiener process $\hat{W}^{i}=$ $\left\{\hat{W}_{\tau^{i}}^{i}, \tau^{i} \geq 0\right\}$ in $\tau^{i}$-time. This is a BESQ process with dimension

$$
\mathfrak{n}_{i}=\frac{3-2 \beta_{i}}{1-\beta_{i}}
$$

for $\beta_{i} \neq 1$ and $i \in\{0,1, \ldots, d\}$. It is convenient to introduce the index $\mathfrak{d}_{i}$ of the BESQ process, and its relationship to both the dimension $\mathfrak{n}_{i}$ and the CEV exponent $\beta_{i}$ as

$$
\mathfrak{d}_{i}=\frac{\mathfrak{n}_{i}-2}{2}=\frac{1}{2\left(1-\beta_{i}\right)}
$$


for $\beta_{i} \neq 1$ and $i \in\{0,1, \ldots, d\}$. The most reasonable family of models for the GOP are obtained from the MCEV model when the exponent is restricted to $\beta_{i} \in[0,1)$. This ensures that the BESQ process underlying the GOP remains strictly positive. It also matches key empirical features of equity indices such as the leverage effect discussed in Black (1976). However, when $\beta_{i} \in(1, \infty)$, the underlying BESQ process has dimension $\mathfrak{n}_{i}<2$, and will reach zero with strictly positive probability.

Approaching the problem from a different perspective, Heath \& Platen (2002) formulate an SDE for the MCEV model under a hypothetical risk-neutral measure $P_{i, \theta}$ to match the classical literature. Following identical steps to the real-world case, they find that the GOP is governed by an underlying BESQ process, with dimension $\mathfrak{n}_{i, \theta}$, given as

$$
\mathfrak{n}_{i, \theta}=4-\mathfrak{n}_{i}=\frac{1-2 \beta_{i}}{1-\beta_{i}}
$$

for $\beta_{i} \neq 1$ and $i \in\{0,1, \ldots, d\}$. Comparison between the two BESQ process dimensions (3.10) and (3.12) leads to the conclusion that the candidate riskneutral measure $P_{i, \theta}$ under the MCEV model for $\beta_{i} \neq 1$ is never equivalent to the real-world measure $P$. The non-equivalence between the real-world and candidate risk-neutral measures has now become an accepted feature of CEV models, noted previously in Lewis (2000), Delbaen \& Shirakawa (2002) and Heston, Loewenstein \& Willard (2007).

Now we introduce the transition density of $\mathfrak{p}_{\mathfrak{n}_{i}}\left(\tau_{t}^{i}, x_{t}^{i} ; \tau_{\bar{T}}^{i}, x_{\bar{T}}^{i}\right)$ for a BESQ process $X^{i}=\left\{X_{\tau^{i}}^{i}, \tau^{i} \in\left[\tau_{0}^{i}, \tau_{\bar{T}}^{i}\right]\right\}$ with the dimension $\mathfrak{n}_{i}>2$, to move from $x_{t}^{i}=X_{\tau_{t}^{i}}^{i}$ at time $\tau_{t}^{i}$ to $x_{\bar{T}}^{i}=X_{\tau_{\bar{T}}^{i}}^{i}$ at time $\tau_{\bar{T}}^{i}>\tau_{t}^{i}$ as

$$
\mathfrak{p}_{\mathfrak{n}_{i}}\left(\tau_{t}^{i}, x_{t}^{i} ; \tau_{\bar{T}}^{i}, x_{\bar{T}}^{i}\right)=\frac{1}{2\left(\tau_{\bar{T}}^{i}-\tau_{t}^{i}\right)}\left(\frac{x_{\bar{T}}^{i}}{x_{t}^{i}}\right)^{\frac{\mathfrak{o}_{i}}{2}} \exp \left\{-\frac{x_{t}^{i}+x_{\bar{T}}^{i}}{2\left(\tau_{\bar{T}}^{i}-\tau_{t}^{i}\right)}\right\} I_{\mathfrak{d}_{i}}\left(\frac{\sqrt{x_{t}^{i} x_{\bar{T}}^{i}}}{\tau_{\bar{T}}^{i}-\tau_{t}^{i}}\right)
$$

for $\tau_{t}^{i} \in\left[\tau_{0}^{i}, \tau_{\bar{T}}^{i}\right]$ and $i \in\{0,1, \ldots, d\}$. Note the inclusion of the modified Bessel function of the first kind $I_{\mathfrak{d}_{i}}$, defined in Appendix A as relation (A.3). It has been common practise in most $\mathrm{CEV}$ related research to provide the underlying transition density function, although this has been achieved with varying degrees of success because of a number of typographical errors, as noted by Shaw (1998). The transition density for the MCEV case, as provided by Heath \& Platen (2002), also has a typographical error within the exponential term that we remedy below. Hence, the transition density $\mathfrak{q}_{\mathfrak{n}_{i}}\left(t, z_{t}^{i} ; \bar{T}, z_{\bar{T}}^{i}\right)$ for a square root process $Z^{i}=\left\{Z_{t}^{i}, t \in[0, \bar{T}]\right\}$ with the dimension $\mathfrak{n}_{i}>2$, to move from $z_{t}^{i}=Z_{t}^{i}$ at time $t$ to $z_{\bar{T}}^{i}=Z_{\bar{T}}^{i}$ at time $\bar{T}>t$ can be shown via (3.6), (3.7), (3.8) and (3.13) 
to take the form

$$
\begin{aligned}
\mathfrak{q}_{\mathfrak{n}_{i}}\left(t, z_{t}^{i} ; \bar{T}, z_{\bar{T}}^{i}\right) & =\frac{1}{A_{\bar{T}}^{i}} \mathfrak{p}_{\mathfrak{n}_{i}}\left(\tau_{t}^{i}, \frac{z_{t}^{i}}{A_{t}^{i}} ; \tau_{\bar{T}}^{i}, \frac{z_{\bar{T}}^{i}}{A_{\bar{T}}^{i}}\right) \\
& =\frac{1}{2\left(\tau_{\bar{T}}^{i}-\tau_{t}^{i}\right) A_{\bar{T}}^{i}}\left(\frac{z_{\bar{T}}^{i} / A_{\bar{T}}^{i}}{z_{t}^{i} / A_{t}^{i}}\right)^{\frac{\mathfrak{o}_{i}}{2}} \exp \left\{-\frac{\frac{z_{t}^{i}}{A_{t}^{i}}+\frac{z_{\bar{T}}^{i}}{A_{\bar{T}}^{i}}}{2\left(\tau_{\bar{T}}^{i}-\tau_{t}^{i}\right)}\right\} I_{\mathfrak{d}_{i}}\left(\frac{\sqrt{\frac{z_{t}^{i} z_{\bar{T}}^{i}}{A_{t}^{i} A_{\bar{T}}^{i}}}}{\tau_{\bar{T}}^{i}-\tau_{t}^{i}}\right)
\end{aligned}
$$

for $t \in[0, \bar{T}]$ and $i \in\{0,1, \ldots, d\}$.

\section{Interest Rate Term Structure}

Traditional approaches to CEV modelling focus on stock prices and say little, if anything at all about the pricing of zero-coupon bonds, let alone attempting to price the two financial instruments consistently. However, the benchmark approach produces a consistent price system for all financial quantities via their relationship to the GOP. A simple calculation under the benchmark approach is to obtain the real-world price of a zero-coupon bond. Here, we will observe our first explicit difference between real-world and putative risk-neutral prices under the MCEV model.

The real-world price of a zero-coupon bond $P^{i}(t, \bar{T})$ in the $i$ th currency at time $t$ with fixed maturity $\bar{T} \in[0, T]$ is defined as the value of a payoff of one unit of the $i$ th currency and is found using the real-world pricing formula (2.7) as

$$
P^{i}(t, \bar{T})=E\left[\frac{S_{t}^{i, \delta_{*}}}{S_{\bar{T}}^{i, \delta_{*}}} \mid \mathcal{A}_{t}\right]
$$

for $t \in[0, \bar{T}]$ and $i \in\{0,1, \ldots, d\}$. Note that $P^{i}(\bar{T}, \bar{T})=1$. Throughout this paper we will assume that the $i$ th short rate process $r^{i}$ and the discounted GOP process $\bar{S}^{i, \delta_{*}}$ are independent. This assumption allows us to decompose the realworld zero-coupon bond price $P^{i}(t, \bar{T})$ in the following multiplicative way

$$
P^{i}(t, \bar{T})=E\left[\frac{\bar{S}_{t}^{i, \delta_{*}}}{\bar{S}_{\bar{T}}^{i, \delta_{*}}} \frac{B_{t}^{i}}{B_{\bar{T}}^{i}} \mid \mathcal{A}_{t}\right]=M_{\bar{T}}^{i}(t) G_{\bar{T}}^{i}(t)
$$

where the discounted GOP contribution to the zero-coupon bond price is

$$
M_{\bar{T}}^{i}(t)=E\left[\frac{\bar{S}_{t}^{i, \delta_{*}}}{\bar{S}_{\bar{T}}^{i, \delta_{*}}} \mid \mathcal{A}_{t}\right]=E\left[\frac{\Lambda_{\bar{T}}^{i, \theta}}{\Lambda_{t}^{i, \theta}} \mid \mathcal{A}_{t}\right]
$$

and the short rate contribution to the zero-coupon bond price is

$$
G_{\bar{T}}^{i}(t)=E\left[\frac{B_{t}^{i}}{B_{\bar{T}}^{i}} \mid \mathcal{A}_{t}\right]
$$


for $t \in[0, \bar{T}]$ and $i \in\{0,1, \ldots, d\}$. Empirical evidence to support the assumption of independence between the driving process of the discounted GOP and the short rate is provided in Miller \& Platen (2005).

To simplify calculations and exposition we assume that the short rate is constant for the remainder of the paper, hence $r_{t}^{i}=r_{i}$ for all $t \in[0, T]$ and $i \in\{0,1, \ldots, d\}$. This will focus attention on the differences that arise from the discounted GOP contribution to the zero-coupon bond given in (4.3). The proof of the following lemma for the price of a zero-coupon bond under the MCEV model is contained in Appendix B. It requires knowledge of the central chi-square distribution function $\chi^{2}(\cdot ; \nu)$ where $\nu$ represents the degrees of freedom defined in Johnson, Kotz \& Balakrishnan (1994).

Lemma 4.1 The real-world price of a zero-coupon bond $P^{i}(t, \bar{T})$ in the ith currency calculated at time $t$ with maturity $\bar{T}$ under the MCEV model is

$$
P^{i}(t, \bar{T})=\exp \left\{-r_{i}(\bar{T}-t)\right\} \chi^{2}\left(\mathfrak{L}_{\bar{T}}^{i} ; \frac{1}{1-\beta_{i}}\right)
$$

where

$$
\mathfrak{L}_{\bar{T}}^{i}=\frac{2 r_{i}}{\left|\theta_{t}^{i}\right|^{2}\left(1-\beta_{i}\right)\left[1-\exp \left\{-2\left(1-\beta_{i}\right) r_{i}(\bar{T}-t)\right\}\right]}
$$

for $t \in[0, \bar{T}]$ and $i \in\{0,1, \ldots, d\}$.

The zero-coupon bond price decomposition of (4.2) applies to Lemma 4.1. Hence it can be deduced from (4.3) and (4.4) that for the MCEV model

$$
\begin{aligned}
& G_{\bar{T}}^{i}(t)=E\left[\frac{B_{t}^{i}}{B_{\bar{T}}^{i}} \mid \mathcal{A}_{t}\right]=\exp \left\{-r_{i}(\bar{T}-t)\right\} \\
& M_{\bar{T}}^{i}(t)=E\left[\frac{\bar{S}_{t}^{i, \delta_{*}}}{\bar{S}_{\bar{T}}^{i, \delta_{*}}} \mid \mathcal{A}_{t}\right]=\chi^{2}\left(\mathfrak{L}_{\bar{T}}^{i} ; \frac{1}{1-\beta_{i}}\right)
\end{aligned}
$$

for $t \in[0, \bar{T}]$ and $i \in\{0,1, \ldots, d\}$. The discounted GOP contribution to the zero-coupon bond price given in (4.8), can also be proven independently using the transition density associated with the time-transformed BESQ process, or by equivalent methods. In fact, this result expressed in terms of the incomplete gamma function ratio [see Abramowitz \& Stegun (1970)] appears in Feller (1951), Shaw (1998) and Lewis (2000), but not in the context of pricing zero-coupon bonds. On the other hand, Heston, Loewenstein \& Willard (2007) find a similar quantity when pricing a zero-coupon bond under the Cox, Ingersoll \& Ross (1985) model. However, none of the above authors use the probabilistic interpretation of the central chi-square distribution proposed above.

Two interesting points arise from the above result. First, we recall from (4.3) that $M_{\bar{T}}^{i}(\cdot)$ represents both the discounted GOP contribution to the zero-coupon 
bond and the expected value of the Radon-Nikodym derivative for the putative risk-neutral measure. Then by (4.6) and (4.8), one observes that $M_{\bar{T}}^{i}(t) \in[0,1]$ for $t \in[0, \bar{T}]$ and $i \in\{0,1, \ldots, d\}$, as it is described by a distribution function. Since the Radon-Nikodym derivative $\Lambda^{i, \theta}$ under the above MCEV model is a non-negative strict $(\mathcal{A}, P)$-local martingale, it is a strict $(\mathcal{A}, P)$-supermartingale. This property explains the differences between the real-world and candidate riskneutral zero-coupon bond prices under the MCEV model. Furthermore, one immediately observes that the key assumption of the risk-neutral approach, namely the martingale property of $\Lambda^{i, \theta}$, is not satisfied. Thus under the MCEV model, not only are the real-world and candidate risk-neutral measures not equivalent, but the corresponding Radon-Nikodym derivative is not an $(\underline{\mathcal{A}}, P)$-martingale either. Thus the MCEV model is an example of a viable financial market, as discussed in Loewenstein \& Willard (2000), where the traditional NFLVR condition of no-arbitrage specified by Delbaen \& Schachermayer (1994), is too strong. Hence a more general methodology such as the benchmark approach discussed in Platen (2002) and Platen \& Heath (2006) is more suitable.

The second observation comes from examining a graphical depiction of $M_{T}^{i}(0)$ for $\bar{T}>0$. In Figure 1 a chart is given for the exponent range $\beta \in[0,1)$ and the maturity range $\bar{T} \in[0,100]$. We use the input parameters of: $t=0 ; r_{i}=0.05$; and $\left|\theta_{0}^{i}\right|=0.25$. Inspection of Figure 1 reveals that $M_{\bar{T}}^{i}(0)$ remains at unity under the case of $\beta_{i}=1$, which is equivalent to the classical Black-ScholesMerton model. However, for exponent levels of $\beta_{i} \in[0,1)$, Figure 1 shows the strict supermartingale property of $M_{\bar{T}}^{i}(0)$.

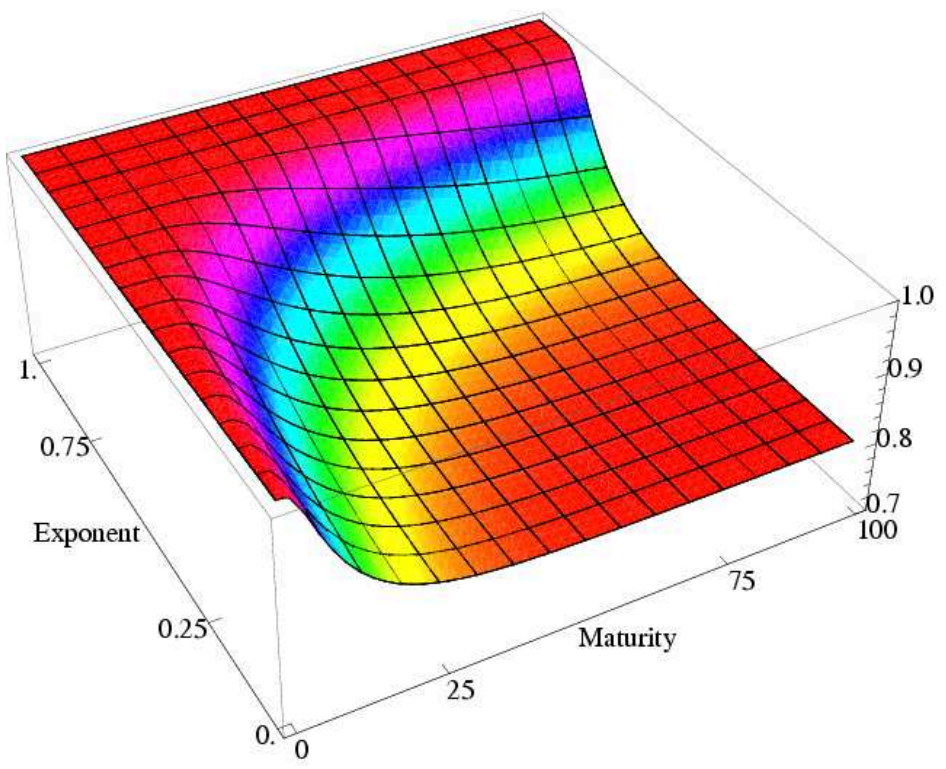

Figure 1: Discounted GOP contribution $M_{\bar{T}}^{i}(0)$ with $\beta_{i} \in[0,1)$ for $\bar{T} \in[0,100]$.

Figure 1 also reveals the fact that $M_{\bar{T}}^{i}(0)$ appears to approach a constant for large 
maturity times $\bar{T} \rightarrow \infty$. In particular, one finds that

$$
M_{\infty}^{i}(0)=\lim _{\bar{T} \rightarrow \infty} M_{\bar{T}}^{i}(0)=\chi^{2}\left(\frac{2 r_{i}}{\left|\theta_{0}^{i}\right|^{2}\left(1-\beta_{i}\right)} ; \frac{1}{1-\beta_{i}}\right)
$$

for $i \in\{0,1, \ldots, d\}$. In addition, if we impose reasonable restrictions on the parameter inputs, such as $r_{i}>0,\left|\theta_{0}^{i}\right|>0$ and $\beta_{i} \in[0,1)$ with economically sensible upper bounds for both $r_{i}$ and $\left|\theta_{0}^{i}\right|$, then $M_{\infty}^{i}(0)$ will be strictly positive. Therefore, the real-world price of a zero-coupon bond under the MCEV model possesses lower and upper bounds, these being

$$
\chi^{2}\left(\frac{2 r_{i}}{\left|\theta_{t}^{i}\right|^{2}\left(1-\beta_{i}\right)} ; \frac{1}{1-\beta_{i}}\right) G_{\bar{T}}^{i}(t) \leq P^{i}(t, \bar{T}) \leq G_{\bar{T}}^{i}(t)
$$

for $t \in[0, \bar{T}]$ and $i \in\{0,1, \ldots, d\}$. The upper bound $G_{\bar{T}}^{i}(t)$ is the putative risk-neutral zero-coupon bond price. A graphical depiction of the relationships between the three quantities in (4.10) is shown in Figure 2 using the parameters: $t=0 ; r_{i}=0.05 ;\left|\theta_{0}^{i}\right|=0.25 ;$ and $\beta_{i}=0.5$ for the maturity range $\bar{T} \in[0,100]$. The obvious implication of this result is that the difference between real-world and putative risk-neutral bond prices under the MCEV model will be limited in the manner described above.

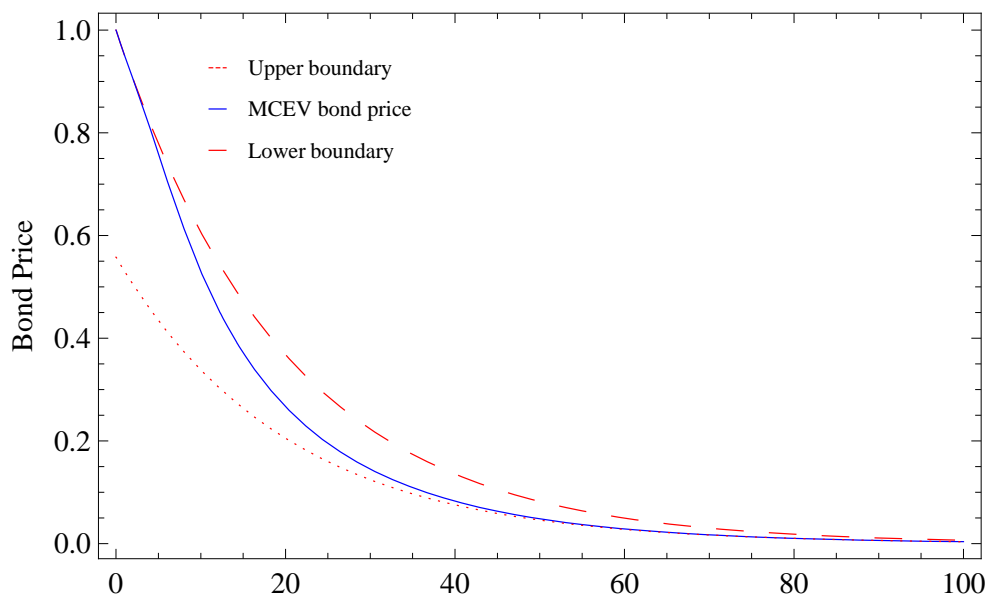

Figure 2: Upper and lower bounds for $P^{i}(0, \bar{T})$ for $\bar{T} \in[0,100]$.

By referring to Heston, Loewenstein \& Willard (2007) a money market bubble is allegedly at play and a weak form of arbitrage seems to arise. To exploit this form of arbitrage the investor has to violate the non-negative wealth constraint that the benchmark approach employs, see Platen (2002), which coincides with the one used in Heston, Loewenstein \& Willard (2007). This means temporary losses are likely before an ultimate profit can be generated from such weak form of arbitrage. 
Given zero-coupon bond prices we can also calculate the instantaneous forward rate $f^{i}(t, \bar{T})$ at time $t$ for the maturity date $\bar{T} \in[0, T]$ in the $i$ th currency as

$$
f^{i}(t, \bar{T})=-\frac{\partial}{\partial \bar{T}} \ln \left[P^{i}(t, \bar{T})\right]
$$

for all $t \in[0, \bar{T}]$ and $i \in\{0,1, \ldots, d\}$. Therefore, in the case when the zerocoupon bond price can be decomposed into the product (4.2), then the forward rate (4.11) takes the form

$$
f^{i}(t, \bar{T})=m_{\bar{T}}^{i}(t)+g_{\bar{T}}^{i}(t)
$$

where the discounted GOP contribution to the forward rate is

$$
m_{\bar{T}}^{i}(t)=-\frac{\partial}{\partial \bar{T}} \ln \left[M_{\bar{T}}^{i}(t)\right]
$$

and the short rate contribution to the forward rate is

$$
g_{\bar{T}}^{i}(t)=-\frac{\partial}{\partial \bar{T}} \ln \left[G_{\bar{T}}^{i}(t)\right]
$$

for $t \in[0, \bar{T}]$ and $i \in\{0,1, \ldots, d\}$.

Since we assume that the short rate is assumed to be constant, the short rate contribution to the forward rate is trivially calculated to be the short rate itself. In contrast to the classical Black-Scholes-Merton type model, the discounted GOP contribution to the forward rate, denoted by $m_{\bar{T}}^{i}(t)$, is non-zero since the Radon-Nikodym derivative $\Lambda^{i, \theta}$ is a strict $(\underline{\mathcal{A}}, P)$-supermartingale. Specifically, we calculate its value using (4.8) and (4.13) to be

$$
m_{\bar{T}}^{i}(t)=\frac{\left(\frac{\mathcal{L}_{T}^{i}}{2}\right)^{1+\frac{1}{2\left(1-\beta_{i}\right)}}\left|\theta_{t}^{i}\right|^{2}\left(1-\beta_{i}\right) \exp \left\{-2\left(1-\beta_{i}\right) r_{i}(\bar{T}-t)-\frac{\mathfrak{L}_{T}^{i}}{2}\right\}}{\Gamma\left(1+\frac{1}{2\left(1-\beta_{i}\right)}\right) \chi^{2}\left(\mathfrak{L}_{\bar{T}}^{i} ; \frac{1}{1-\beta_{i}}\right)}
$$

for all $t \in[0, \bar{T}]$ and $i \in\{0,1, \ldots, d\}$. In essence $m_{\bar{T}}^{i}(t)$ is a transformation of the rate of change of $M_{\bar{T}}^{i}(t)$ with respect to maturity. Figure 3 illustrates the general shape of $m_{\bar{T}}^{i}(0)$ for the parameters: $t=0 ;\left|\theta_{0}^{i}\right|=0.25 ; r_{i}=0.05 ; \beta_{i} \in[0,1)$ and $\bar{T} \in[0,30]$. The resulting contribution to the forward rate is hump-shaped, and as with all aspects of the MCEV model, depends heavily on the value of the exponent $\beta_{i}$. For lower levels of the CEV exponent $\beta_{i}$, the hump shape is more peaked and occurs earlier in the term structure. Also note that $m_{\infty}^{i}(0)=0$.

Both forward rates and zero-coupon bond prices under the MCEV model are reasonably sensitive to changes in the initial level of the volatility of the GOP. The economic interpretation of this relationship between $\left|\theta_{t}^{i}\right|$ and $m_{\bar{T}}^{i}(t)$ is interesting and intuitive. The volatility of the GOP primarily affects medium- to longterm maturities of the yield curve, as can be observed in both Figures 1 and 


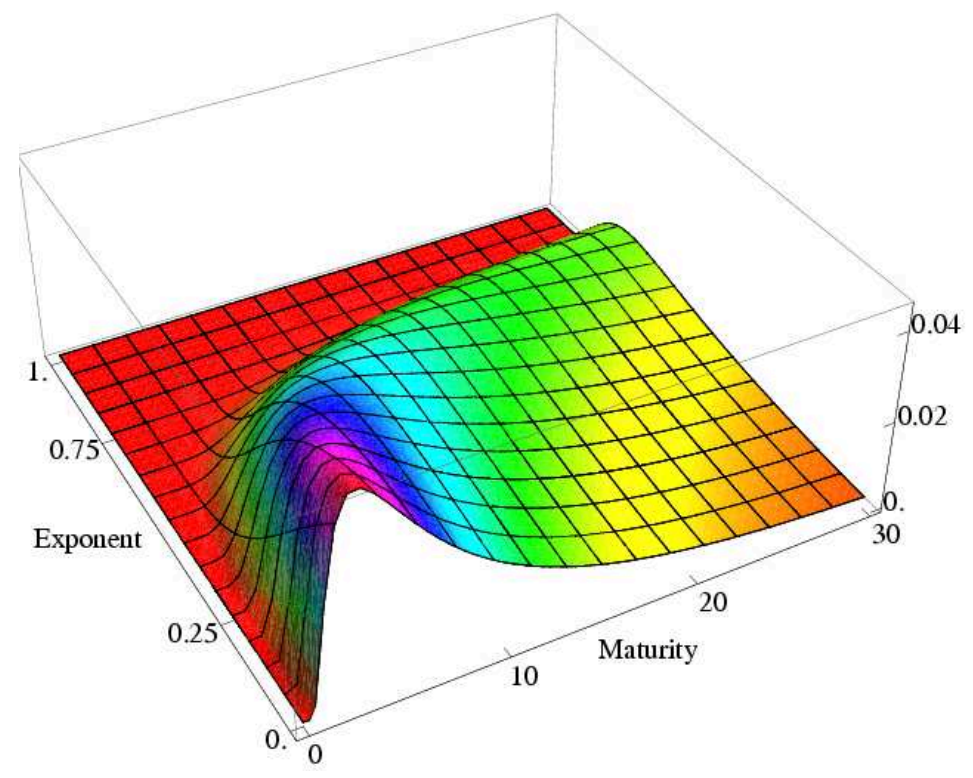

Figure 3: Discounted GOP contribution to the forward rate $m_{\bar{T}}^{i}(0)$ for $\beta_{i} \in[0,1)$ and $\bar{T} \in[0,30]$.

3. This accords well with both economic theory and practice. While shortdated interest rate instruments are primarily influenced by the monetary policy specific to that currency, longer dated maturities of the yield curve are influenced by many factors, most notably supply and demand, as well as local and global economic conditions. Therefore, the economic drivers of both equity markets and the long-end of the yield curve are correlated. Since the volatility of the GOP is a good proxy for the volatility of a well-diversified equity index, it can also be interpreted as the volatility, or general level of uncertainty of underlying economic conditions. Furthermore, the relationship between general market volatility and long-term interest rates is usually directional. In periods of increased uncertainty, volatility increases, and hence the 'risk premium' component of long-term yields must also increase to compensate for the increased level of uncertainty. Such a directional relationship between $\left|\theta_{t}^{i}\right|$ and $m_{\bar{T}}^{i}(t)$ is evident within the given MCEV model, as can be seen in Figure 4 below. A corresponding inverse relationship exists between $\left|\theta_{t}^{i}\right|$ and $M_{\bar{T}}^{i}(t)$. The input parameters used in Figure 4 were: $t=0$; $r_{i}=0.05$; and the CEV exponent values $\beta_{i} \in\{0.0,0.25,0.5,0.75\}$. Interestingly, we see that the level of initial GOP volatility has no impact on the forward rate until a 'threshold' volatility level has been reached, which is also intuitive. More generally, one observes from Figure 4 that the magnitude and placement of the hump within the forward rate curve varies with the initial level of GOP volatility. 

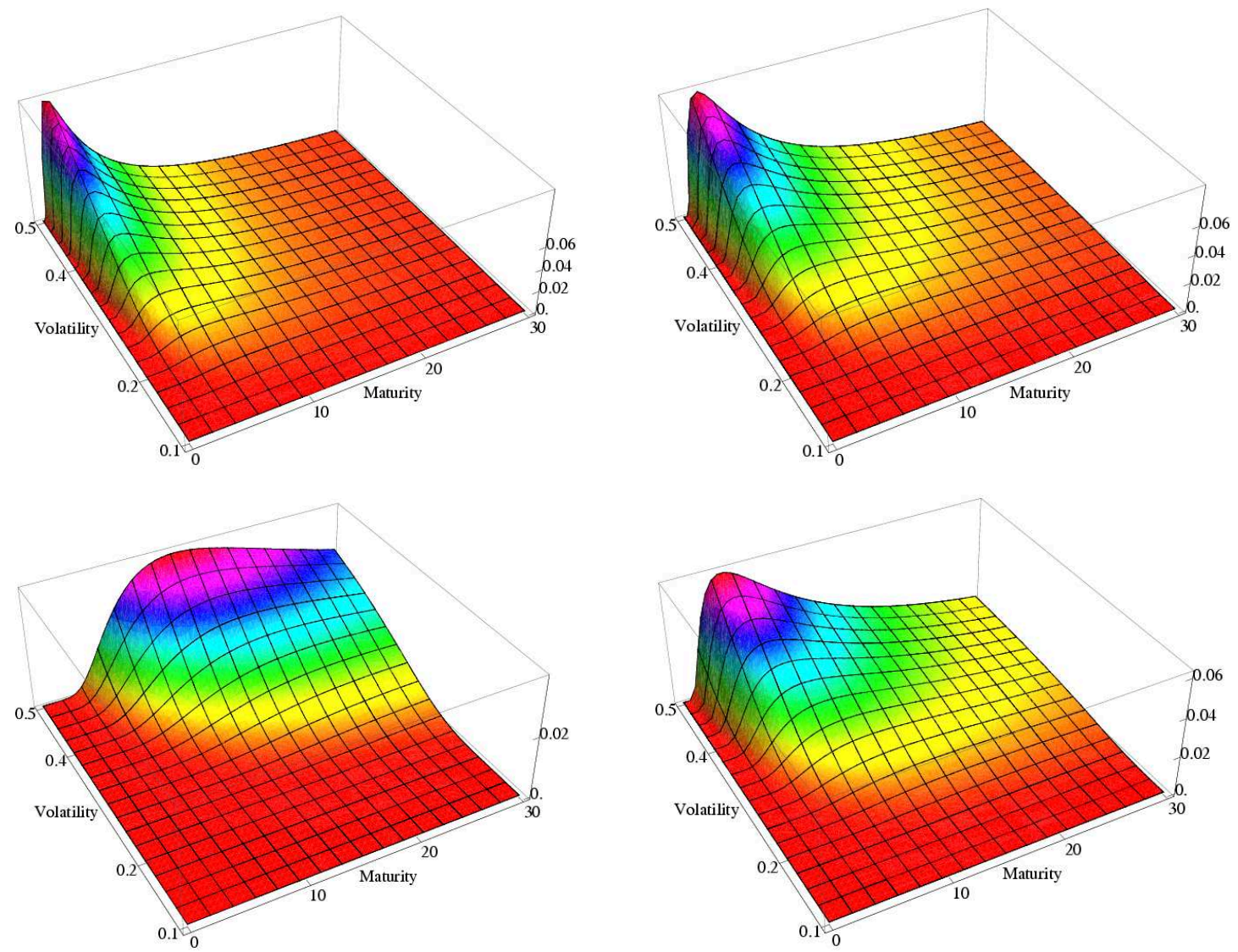

Figure 4: $m_{\bar{T}}^{i}(0)$ for $\left|\theta_{0}^{i}\right| \in[0.05,0.50]$ and $\bar{T} \in[0,30]$ with $\beta_{i} \in$ $\{0.0,0.25,0.5,0.75\}$, clockwise.

\section{Options on the GOP}

Below we provide new analytic formulae for European call and put options on the GOP in terms of central and non-central chi-square distributions. The following results significantly reduce the computational burden of examining implied volatility surfaces from previous numerical techniques, such as those employed by Heath \& Platen (2002). These formulae also contribute to the ongoing discussion on the limitations of risk-neutral pricing. In addition to previous notation, we require the non-central chi-square distribution function $\chi^{2}(\cdot ; \nu, \lambda)$ with degrees of freedom $\nu$ and non-centrality parameter $\lambda$.

Lemma 5.1 The real-world prices of call and put options on the GOP in the ith currency at time $t$ with expiry $\bar{T}$ and strike price $K$ under the MCEV model 
are

$$
\begin{aligned}
c_{\bar{T}, K, S^{i, \delta_{*}}}^{i}(t) & =S_{t}^{i, \delta_{*}}\left[1-\chi^{2}\left(\tilde{\mathfrak{u}}_{\bar{T}}^{i} ; \frac{3-2 \beta_{i}}{1-\beta_{i}}, \mathfrak{L}_{\bar{T}}^{i}\right)\right] \\
& -K \exp \left\{-r_{i}(\bar{T}-t)\right\} \chi^{2}\left(\mathfrak{L}_{\bar{T}}^{i} ; \frac{1}{1-\beta_{i}}, \tilde{\mathfrak{u}}_{\bar{T}}^{i}\right) \\
p_{\bar{T}, K, S^{i}, \delta_{*}}^{i}(t) & =-S_{t}^{i, \delta_{*}} \chi^{2}\left(\tilde{\mathfrak{u}}_{\bar{T}}^{i} ; \frac{3-2 \beta_{i}}{1-\beta_{i}}, \mathfrak{L}_{\bar{T}}^{i}\right)+K \exp \left\{-r_{i}(\bar{T}-t)\right\} \\
& \cdot\left[\chi^{2}\left(\mathfrak{L}_{\bar{T}}^{i} ; \frac{1}{1-\beta_{i}}\right)-\chi^{2}\left(\mathfrak{L}_{\bar{T}}^{i} ; \frac{1}{1-\beta_{i}}, \tilde{\mathfrak{u}}_{\bar{T}}^{i}\right)\right]
\end{aligned}
$$

where

$$
\tilde{\mathfrak{u}}_{\bar{T}}^{i}=\frac{2 r_{i}\left(S_{t}^{i, \delta_{*}} / K\right)^{2\left(\beta_{i}-1\right)}}{\left|\theta_{t}^{i}\right|^{2}\left(1-\beta_{i}\right)\left[\exp \left\{2\left(1-\beta_{i}\right) r_{i}(\bar{T}-t)\right\}-1\right]}
$$

for $t \in[0, \bar{T}], i \in\{0,1, \ldots, d\}$ with $\mathfrak{L}_{\bar{T}}^{i}$ as per definition $(4.6)$.

Call options on the GOP yield exactly the same prices under either the real-world or candidate risk-neutral measures, however the prices of put options on the GOP differ. The proof of the lemma is provided in Appendix B.

The calculation of contingent claims under the real-world measure involves taking the expectation of the inverse of the GOP at some future point in time. In the case of the given MCEV model, this translates into taking the expectation of the inverse of a scaled and time-transformed BESQ process with dimension $\mathfrak{n}_{i}>2$. It was shown in Yor (1992) that this is equivalent to taking the expectation of a BESQ process with dimension $\mathfrak{n}_{i, \theta}=\left(4-\mathfrak{n}_{i}\right)<2$ with an absorbing barrier at zero. Therefore, there exists positive probability for the equivalent process to reach zero, impacting real-world contingent claim values calculated under the given MCEV model. This means that all contingent claims with a non-zero payoff at the level zero, such as zero-coupon bonds and put options, will have lower realworld prices compared to corresponding putative risk-neutral prices. Attempting to price such payoffs under the risk-neutral paradigm will lead to higher prices under the MCEV model, and thus incorrect results.

In addition to the price of a call option on the GOP given in Lemma 5.1, we can also derive an alternative representation based on the two-parameter gamma distribution. The corresponding density and complementary distribution function, denoted by $p_{\mathcal{G}}(\cdot / \beta ; \alpha)$ and $\mathcal{G}(\cdot / \beta ; \alpha)$ with shape parameter $\alpha>0$ and scale parameter $\beta>0$, are given in Appendix A as formulae (A.6) and (A.7), respectively. One then obtains the real-world price for a call option as

$$
\begin{aligned}
c_{\bar{T}, K, S^{i, \delta_{*}}}^{i}(t) & =S_{t}^{i, \delta_{*}} \sum_{\ell=0}^{\infty} p_{\mathcal{G}}\left(\frac{\mathfrak{L}_{\bar{T}}^{i}}{2} ; \ell+1\right) \mathcal{G}\left(\frac{\tilde{\mathfrak{u}}_{\bar{T}}^{i}}{2} ; \ell+\frac{1}{2\left(1-\beta_{i}\right)}+1\right) \\
& -\exp \left\{-r_{i}(\bar{T}-t)\right\} \sum_{\ell=0}^{\infty} p_{\mathcal{G}}\left(\frac{\mathfrak{L}_{\bar{T}}^{i}}{2} ; \ell+\frac{1}{2\left(1-\beta_{i}\right)}+1\right) \mathcal{G}\left(\frac{\tilde{\mathfrak{u}}_{\bar{T}}^{i}}{2} ; \ell+1\right)
\end{aligned}
$$


for $t \in[0, \bar{T}], i \in\{0,1, \ldots, d\}$ and $\beta_{i} \in[0,1)$, with (4.6) and (5.3). Comparison of (5.4) to results in Cox (1996), Cox \& Ross (1976), Beckers (1980), Shaw (1998), Delbaen \& Shirakawa (2002) and Schroder (1989) reveal that the price of a call option on the GOP under the MCEV model matches the putative risk-neutral price. The two prices are equal because a call option has a zero payoff for all outcomes below the strike price $K$ at expiry.

We provide in Figure 5 the implied volatility surface obtained for call options on the GOP based on: $t=0 ; S_{0}^{i, \delta_{*}}=2,000 ; r_{i}=0.05 ;\left|\theta_{0}^{i}\right|=0.25 ; \bar{T}=30$; for $\beta_{i} \in\{0.00,0.25,0.50,0.75\}$. Here the rate $r_{\bar{T}}^{i}=\frac{1}{T} \ln \left[P^{i}(0, \bar{T})\right]$ is used as an input when calculating the implied volatility via the Black-Scholes-Merton formula. Otherwise put-call parity will fail. Beginning with the top LHS in Figure 5, one observes for $\beta_{i}=0.00$, a negatively skewed, downward sloping implied volatility surface that is typical for the Gaussian model. Then moving clockwise as the exponent increases to $\beta_{i} \in\{0.25,0.50,0.75\}$, the implied volatility surface flattens out both in terms of skew and slope. Recall for $\beta_{i}=1.00$, a Black-Scholes-Merton model possesses a completely flat implied volatility surface.
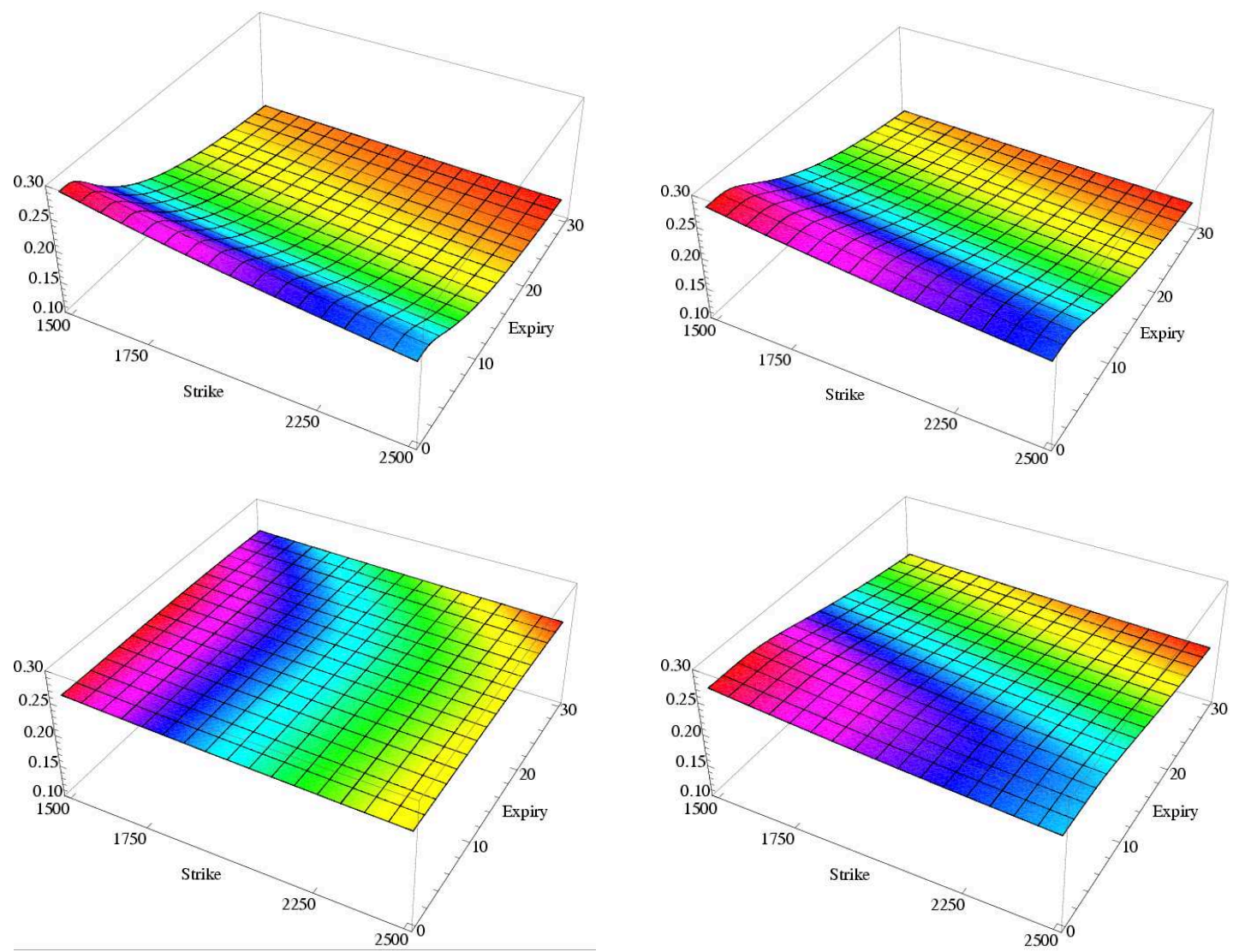

Figure 5: Implied volatility surfaces for options on the GOP with $\bar{T} \in[0,30]$ for $\beta_{i} \in\{0.0,0.25,0.5,0.75\}$, clockwise. 
The MCEV zero-coupon bond price (4.5) is critical in the statement of put-call parity for options on the GOP, given as

$$
c_{\bar{T}, K, S^{i, \delta_{*}}}^{i}(t)+K P^{i}(t, \bar{T})=p_{\bar{T}, K, S^{i, \delta_{*}}}^{i}(t)+S_{t}^{i, \delta_{*}}
$$

for $t \in[0, \bar{T}]$ and $i \in\{0,1, \ldots, d\}$. Heston, Loewenstein \& Willard (2007) argue that for the standard CEV model "one can choose either put-call parity or riskneutral option pricing, but not both". Arguments with the same implications are also provided in Lewis (2000). We can only agree with the first of these conclusions, since under the MCEV model, put-call parity is achieved using realworld pricing, but it is shown to be inconsistent with risk-neutral pricing. Cox \& Hobson (2005) repeat the claim that put-call parity does not hold under riskneutral pricing, but contrary to Heston, Loewenstein \& Willard (2007), do not provide an alternative where it will work. The above discussion clarifies this issue by providing put-call parity under the real-world probability measure using minimal prices for calls, puts and zero-coupon bonds.

Let us relate the results of this section further to the existing CEV literature. Lewis (2000) presents a broad study on option valuation with stochastic volatility, pioneering in its discussion of the failure of the classical risk-neutral pricing methodology. Lewis (2000) discusses a restricted CEV model within a stochastic volatility framework similar to (3.3) under the risk-neutral paradigm. To maintain similar notation to Lewis (2000) we denote the squared GOP volatility process by $v^{i}=\left\{v_{t}^{i}=\left|\theta_{t}^{i}\right|^{2}, t \in[0, T]\right\}$ for $i \in\{0,1, \ldots, d\}$. Upon re-arranging (3.1) we can write the GOP as

$$
S_{t}^{i, \delta_{*}}=\left(\frac{v_{t}^{i}}{\xi_{i}^{2}}\right)^{\frac{1}{2\left(\beta_{i}-1\right)}}=\left(\frac{\xi_{i}^{2}}{v_{t}^{i}}\right)^{\frac{1}{2\left(1-\beta_{i}\right)}}
$$

for $t \in[0, T]$ and $i \in\{0,1, \ldots, d\}$. One can then show via (3.4), (3.8) and (5.6), that the squared volatility of the GOP can be expressed as the inverse of a BESQ process, that is

$$
v_{t}^{i}=\frac{\xi_{i}^{2}}{A_{t}^{i} \bar{Z}_{\tau_{t}}^{i}}
$$

for $t \in[0, T]$ and $i \in\{0,1, \ldots, d\}$. Broadly speaking, the arguments of Lewis (2000) are as follows. Using the test for explosions in Feller (1951), one finds that the squared GOP volatility (3.3) will reach infinity in finite time with strictly positive probability. This is because when the exponent falls within the range $\beta_{i} \in(-\infty, 1)$, the BESQ process in (5.7) will have a risk-neutral dimension $\mathfrak{n}_{i, \theta}=\left(4-\mathfrak{n}_{i}\right)<2$ that reaches zero with strictly positive probability. The natural extension of this fact and (5.6) is that the GOP under the putative risk-neutral measure will reach zero with strictly positive probability, and for the solution to the SDE (3.2) to hold, zero must be an absorbing rather than a reflecting barrier. Hence it is the explosion of the squared volatility of the GOP that leads to an 
'explosion probability adjustment', which achieves corrections equivalent to our results. The work of Lewis (2000) is systematic, however because it assumes that an equivalent risk-neutral probability measure exists, the adjustment appears to be ad-hoc. For example, it is shown that for put-call parity to hold, the 'explosion probability adjustment' is required, but the important link to the prices of zerocoupon bonds is not explored. The adjustments suggested in Lewis (2000) are a straightforward consequence of the benchmark approach.

Heston, Loewenstein \& Willard (2007) also remain within the risk-neutral framework, and therefore produce similar results to Lewis (2000). They begin a discussion on the standard CEV model by specifying an SDE for a stock price under an assumed risk-neutral measure, with the exponent range $\beta_{i} \in[1, \infty)$. In contrast, we study the GOP under the real-world probability, and hence are careful when making comparisons. For the GOP, when $\beta_{i}>1$ the underlying BESQ process will remain strictly positive under the risk-neutral measure, since it has dimension $\mathfrak{n}_{i, \theta}=\left(4-\mathfrak{n}_{i}\right) \geq 2$. However, this implies real-world dynamics for the underlying BESQ process with dimension $\mathfrak{n}_{i}<2$, and thus absorption at zero with strictly positive real-world probability. Therefore Heston, Loewenstein \& Willard (2007) are following the specification of Engel \& MacBeth (1982), where the volatility increases for higher levels of the underlying, and vice-versa. This is contrary to the observed leverage effect documented by Black (1976).

The importance of the work of Heston, Loewenstein \& Willard (2007) is their aim to bridge the gap between mathematics and economic understanding, as in the previous work of Loewenstein \& Willard (2000). They provide three conditions that must be satisfied such that 'asset pricing bubbles' will not exist. The first is that the market price of risk must be finite, while the second is that the candidate Radon-Nikodym derivative, must be an $(\mathcal{A}, P)$-martingale. The third condition amounts to the existence of an equivalent risk-neutral local martingale measure $P_{i, \theta}$ under which the discounted underlying is an $\left(\underline{\mathcal{A}}, P_{i, \theta}\right)$-martingale. Under the benchmark approach, the market price of risk is the volatility of the GOP. The second and third conditions of Heston, Loewenstein \& Willard (2007) are not required. Ultimately the question of 'asset pricing bubbles' amounts to whether or not the boundaries at zero and infinity can be reached. The nature of the solutions obtained are determined by whether these boundaries are reflecting or absorbing, as described within the framework of Feller (1951). The advantage of using the benchmark approach is that real-world pricing will always recover the minimal price and does not require an examination of the boundaries at zero and infinity. However, other pricing rules can only generate non-negative replicating price processes that are greater than or equal to those derived using the real-world pricing formula (2.7) due to the supermartingale property of all non-negative benchmarked portfolios. 


\section{Options on an Exchange Price}

Since the GOP value is effectively modelled as a non-central chi-square random variable under the MCEV model, an exchange price (2.3) is modelled as a ratio of non-central chi-square random variables. By the assumption of independence between different GOP denominations, an exchange price will be given in terms of doubly non-central beta random variables. Hence we introduce the doubly non-central beta distribution function, denoted by $\mathcal{I}\left(\cdot ; \nu_{i}, \nu_{j} ; \lambda_{i}, \lambda_{j}\right)$ with the two degrees of freedom parameters $\nu_{i}, \nu_{j}$ and the two non-centrality parameters $\lambda_{i}, \lambda_{j}$, as defined in Johnson, Kotz \& Balakrishnan (1995). Second, the CEV exponent $\beta_{i}=\beta$ is assumed to take identical values in each GOP denomination. Then the index $\mathfrak{d}_{i}=\mathfrak{d}$ of each of the BESQ processes underlying the GOP must also be identical by (3.10). Third, we employ the symmetry property of the modified Bessel function of the first kind (A.3), such that $I_{\mathfrak{d}}(\cdot)=I_{-\mathfrak{d}}(\cdot)$ for all $\mathfrak{d} \in\{\ldots,-2,-1,0,+1,+2, \ldots\}$, which restricts the CEV exponent to $\beta \in$ $\left\{\frac{1}{2}, \frac{3}{4}, \frac{5}{6}, \ldots\right\}$. We now evaluate exchange price options under the given constraints.

Lemma 6.1 The real-world prices of call and put options on an exchange price in the ith currency at time $t$ with expiry $\bar{T}$ and strike price $K$ under the MCEV model for exponent $\beta \in\left\{\frac{1}{2}, \frac{3}{4}, \frac{5}{6}, \ldots\right\}$ are

$$
\begin{aligned}
& c_{\bar{T}, K, X^{i, j}}^{i}(t)=-K e^{-r_{i}(\bar{T}-t)}\left[1-\mathcal{I}\left(\frac{d_{i, j}}{1+d_{i, j}} ; \frac{1-2 \beta}{1-\beta}, \frac{3-2 \beta}{1-\beta} ; \mathfrak{L}_{\bar{T}}^{i}, \mathfrak{L}_{\bar{T}}^{j}\right)\right] \\
& +X_{t}^{i, j} e^{-r_{j}(\bar{T}-t)}\left[\mathcal{I}\left(\frac{1 / d_{i, j}}{1+1 / d_{i, j}} ; \frac{1-2 \beta}{1-\beta}, \frac{3-2 \beta}{1-\beta} ; \mathfrak{L}_{\bar{T}}^{j}, \mathfrak{L}_{\bar{T}}^{i}\right)-\mathcal{Q}\left(\frac{\mathfrak{L}_{\bar{T}}^{j}}{2} ; \frac{1}{2(1-\beta)}\right)\right] \\
& p_{\bar{T}, K, X^{i, j}}^{i}(t)=-X_{t}^{i, j} e^{-r_{j}(\bar{T}-t)}\left[1-\mathcal{I}\left(\frac{1 / d_{i, j}}{1+1 / d_{i, j}} ; \frac{1-2 \beta}{1-\beta}, \frac{3-2 \beta}{1-\beta} ; \mathfrak{L}_{\bar{T}}^{j}, \mathfrak{L}_{\bar{T}}^{i}\right)\right] \\
& +K e^{-r_{i}(\bar{T}-t)}\left[\mathcal{I}\left(\frac{d_{i, j}}{1+d_{i, j}} ; \frac{1-2 \beta}{1-\beta}, \frac{3-2 \beta}{1-\beta} ; \mathfrak{L}_{\bar{T}}^{i}, \mathfrak{L}_{\bar{T}}^{j}\right)-\mathcal{Q}\left(\frac{\mathfrak{L}_{\bar{T}}^{i}}{2} ; \frac{1}{2(1-\beta)}\right)\right]
\end{aligned}
$$

where

$$
d_{i, j}=\frac{\left|\theta_{t}^{j}\right|^{2}\left[\exp \left\{2(1-\beta) r_{j}(\bar{T}-t)\right\}-1\right] r_{i}}{\left|\theta_{t}^{i}\right|^{2}\left[\exp \left\{2(1-\beta) r_{i}(\bar{T}-t)\right\}-1\right] r_{j}\left(X_{t}^{i, j} / K\right)^{2(1-\beta)}}
$$

for $t \in[0, \bar{T}]$ and $i, j \in\{0,1, \ldots, d\}$, where $\mathfrak{L}_{\bar{T}}^{k}$ for $k \in\{i, j\}$ equates to (4.6).

One can verify with Lemma 6.1, proven in Appendix B, that put-call parity holds for options on an exchange price when the real-world domestic zero-coupon bond is used to discount the strike price, and the foreign zero-coupon bond in the $j$ th denomination is used to discount the exchange price, that is

$$
c_{\bar{T}, K, X^{i, j}}^{i}(t)+K P^{i}(t, \bar{T})=p_{\bar{T}, K, X^{i, j}}^{i}(t)+X_{t}^{i, j} P^{j}(t, \bar{T})
$$

for $t \in[0, \bar{T}]$ and $i, j \in\{0,1, \ldots, d\}$. 
We provide in Figure 6 implied volatility surfaces for call options on exchange prices under the MCEV model for the exponent levels of $\beta \in\{0.00,0.25,0.50,0.75\}$, clockwise respectively. The remaining input parameters used were: $t=0$; $X_{0}^{i, j}=1.0 ;\left|\theta_{0}^{i}\right|=\left|\theta_{0}^{j}\right|=0.25 ; r_{i}=r_{j}=0.05$; for $\bar{T} \in[0,30]$ in each case. The results show that higher levels of implied volatility are exhibited for greater values of the exponent. Again, one observes the transformation from a downward sloping, skewed volatility surface associated with the Gaussian model of $\beta=0.0$ to the flat volatility surface of the Black-Scholes-Merton model with $\beta=1.0$.
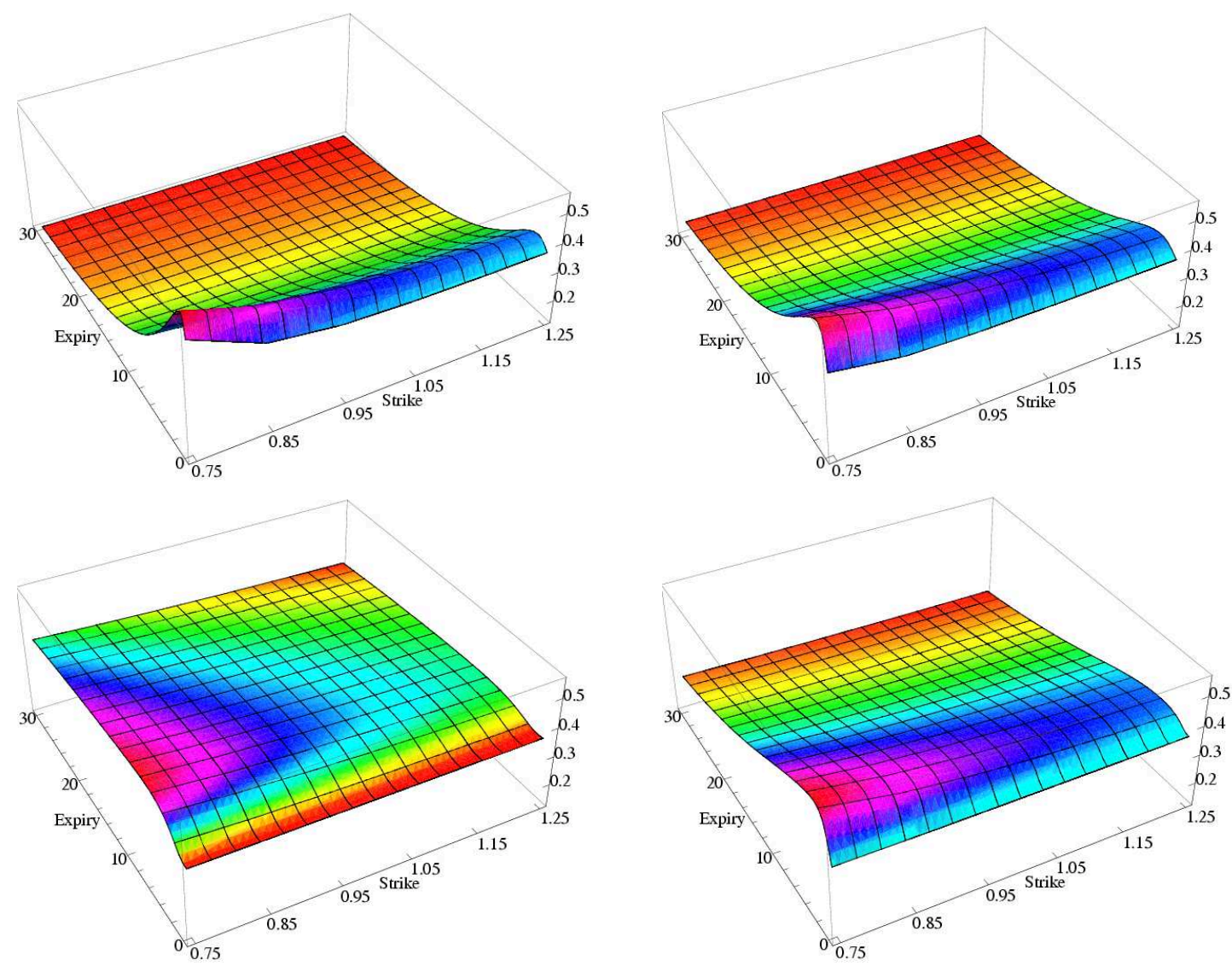

Figure 6: Implied volatility surfaces for exchange price options with $\bar{T} \in[0,30]$ for $\beta \in\{0.0,0.25,0.5,0.75\}$, clockwise.

We remark that by using non-central Wishart distributions, see Bru (1991), for related non-central chi-square random variables one can, in principle, expect an analytic formula for options on exchange prices.

\section{Interest Rate Options}

Finally, we derive real-world prices for basic interest rate options under the given MCEV model. We reiterate the assumptions of a constant short rate, that is 
$r_{t}^{i}=r_{i}$ for all $t \in[0, T]$ and $i \in\{0,1, \ldots, d\}$, and that the volatility of the GOP satisfies relation (3.1). The assumption of a constant short rate is essential to the derivation of the semi-analytic results presented below. Despite the limitations of a constant short rate, the interest rate term structure derived under the MCEV model is useful because of its simplicity, tractability and ability to highlight interesting effects resulting from the volatility of the GOP.

We know that real-world MCEV zero-coupon bond prices are bounded, as previously observed in (4.10). Obviously, bounds for the bond price translate into bounds for forward rates. In particular, when the short rate is constant the forward rate will never fall below the value of the short rate.

In order to determine real-world prices for options on zero-coupon bonds we first introduce the inverse of the regularised incomplete gamma function, denoted by $\mathcal{Q}^{-1}[\cdot ; \nu]$ with $\nu$ degrees of freedom, as defined in Abramowitz \& Stegun (1970). Also note that when $\beta_{i}=0.5$ the following formulae reduce to analytic forms.

Lemma 7.1 The real-world prices of call and put options on a forward zerocoupon bond, $P^{i}(\bar{T}, T)$ with maturity $T \geq \bar{T}$ in the ith currency with strike price $K$ and option expiry $\bar{T}$ under the given $M C E V$ model are

$$
\begin{aligned}
& \boldsymbol{z} \boldsymbol{b} \boldsymbol{c}_{\bar{T}, T, K}^{i}(t)=\left[+e^{-r_{i}(T-t)}-K e^{-r_{i}(\bar{T}-t)}\right] \chi^{2}\left(\mathfrak{L}_{\bar{T}}^{i} ; \frac{1}{1-\beta_{i}}, \tilde{\mathfrak{p}}_{i}\right)-\Xi_{\bar{T}, T, P^{i}(\bar{T}, T)}^{i,+}(t) \\
& \boldsymbol{z} \boldsymbol{b} \boldsymbol{p}_{\bar{T}, T, K}^{i}(t)= \begin{cases}-e^{-r_{i}(T-t)}\left[\chi^{2}\left(\mathfrak{L}_{\bar{T}}^{i} ; \frac{1}{1-\beta_{i}}\right)-\chi^{2}\left(\mathfrak{L}_{\bar{T}}^{i} ; \frac{1}{1-\beta_{i}}, \tilde{\mathfrak{p}}_{i}\right)\right] \\
+K e^{-r_{i}(\bar{T}-t)}\left[\chi^{2}\left(\mathfrak{L}_{T}^{i} ; \frac{1}{1-\beta_{i}}\right)-\chi^{2}\left(\mathfrak{L}_{\bar{T}}^{i} ; \frac{1}{1-\beta_{i}}, \tilde{\mathfrak{p}}_{i}\right)\right] \\
-\Xi_{\bar{T}, T, P}^{i,+}(\bar{T}, T) \\
-P^{i}(t, T)+K P^{i}(t, \bar{T}) & \text { for } K<G_{T}^{i}(\bar{T}) \\
& \text { for } K \geq G_{T}^{i}(\bar{T})\end{cases}
\end{aligned}
$$

where

$$
\Xi_{\bar{T}, T, P^{i}(\bar{T}, T)}^{i,+}(t)=E\left[\frac{S_{t}^{i, \delta_{*}}}{S_{\bar{T}}^{i, \delta_{*}}} \frac{B_{\bar{T}}^{i}}{B_{T}^{i}} \mathcal{Q}\left(\frac{\left(S_{\bar{T}}^{i, \delta_{*}}\right)^{2\left(1-\beta_{i}\right)}}{2 A_{\bar{T}}^{i}\left(\tau_{T}^{i}-\tau_{\bar{T}}^{i}\right)} ; \frac{1}{1-\beta_{i}}\right) \mathbf{1}_{\left\{S_{\bar{T}}^{i, \delta_{*}} \geq \mathfrak{p}_{i}\right\}} \mid \mathcal{A}_{t}\right]
$$

with

$$
\begin{aligned}
\tilde{\mathfrak{p}}_{i} & =\frac{2\left(1-\exp \left\{-2\left(1-\beta_{i}\right) r_{i}(T-\bar{T})\right\}\right)}{\exp \left\{2\left(1-\beta_{i}\right) r_{i}(\bar{T}-t)\right\}-1} \mathcal{Q}^{-1}\left[\frac{G_{T}^{i}(\bar{T})-K}{G_{T}^{i}(\bar{T})} ; \frac{1}{2\left(1-\beta_{i}\right)}\right] \\
\mathfrak{L}_{T}^{i} & =\frac{2 r_{i}}{\left|\theta_{t}^{i}\right|^{2}\left(1-\beta_{i}\right)\left[1-\exp \left\{-2\left(1-\beta_{i}\right) r_{i}(T-t)\right\}\right]}
\end{aligned}
$$

for $0 \leq t \leq \bar{T} \leq T$ and $i \in\{0,1, \ldots, d\}$ with notation (4.5)-(4.7).

The most interesting aspect of Lemma 7.1, also derived in Appendix B, is the price of the put option on a zero-coupon bond. The separation of (7.2) into two cases arises because of the restriction implied by the inverse of the regularised incomplete gamma function within equation (7.4). In the case when this restriction 
is binding, that is $K \geq G_{T}^{i}(\bar{T})$, the put option price reduces to a simple relation involving only zero-coupon bond prices and the strike price. On the other hand, for the general case of a call option on a zero-coupon bond and for the specific case of a put option when $K<G_{T}^{i}(\bar{T})$, numerical evaluation is necessary because the solutions require evaluation of the expectation (7.3). This can be achieved by using the substitution (3.4) and numerical integration of the transition density (3.14).

The results in Lemma 7.1 can also be used to verify the put-call parity relationship for options on zero-coupon bonds, given as

$$
\mathbf{z b c}_{\bar{T}, T, K}^{i}(t)+K P^{i}(t, \bar{T})=\mathbf{z b p}_{\bar{T}, T, K}^{i}(t)+P^{i}(t, T)
$$

for $0 \leq t \leq \bar{T}<T$ and $i \in\{0,1, \ldots, d\}$.

One observes that in the case of a call option on a zero-coupon bond, the realworld MCEV price will always be less than or equal to the corresponding putative risk-neutral price. Therefore risk-neutral prices, for both zero-coupon bonds and call options thereon, form an upper bound for corresponding real-world MCEV prices. Unlike the call option case, the relationship between the risk-neutral and MCEV put option prices is complex and no obvious conclusions seem to emerge from a comparison between the two results.

Finally we provide results obtained for interest rate caps and floors under the real-world MCEV model. Recall that interest rate caps and floors are defined in terms of simple transformations from options on zero-coupon bonds, as described in Brigo \& Mercurio (2005).

In Figure 7 we display the at-the-money (ATM) caplet implied volatility term structure under the MCEV model for $\beta_{i} \in\{0.00,0.25,0.50,0.75\}$, clockwise respectively. Numerical values used were: $t=0 ;\left|\theta_{0}^{i}\right|=0.25 ; r_{i}=0.05$; for $\bar{T}=[0,30]$ in each case. Since we only consider ATM implied volatilities, realworld MCEV interest rate floorlet prices would produce the same results. The key features of Figure 7 are that the hump-shaped caplet volatilities are only significant for medium- and long-term segments of the term structure, and that the magnitude is greater for lower levels of the exponent $\beta_{i}$. This latter feature is consistent with previous results in this paper and emphasizes the fact that the long-dated real-world prices will be less than putative risk-neutral prices.

\section{Conclusion}

New analytic formulae for zero-coupon bonds, options on the GOP, and options on exchange prices have been derived for the MCEV model under the real-world probability measure. Related semi-analytic formulae for options on zero-coupon bonds were also provided. This paper has shown explicitly that real-world and 

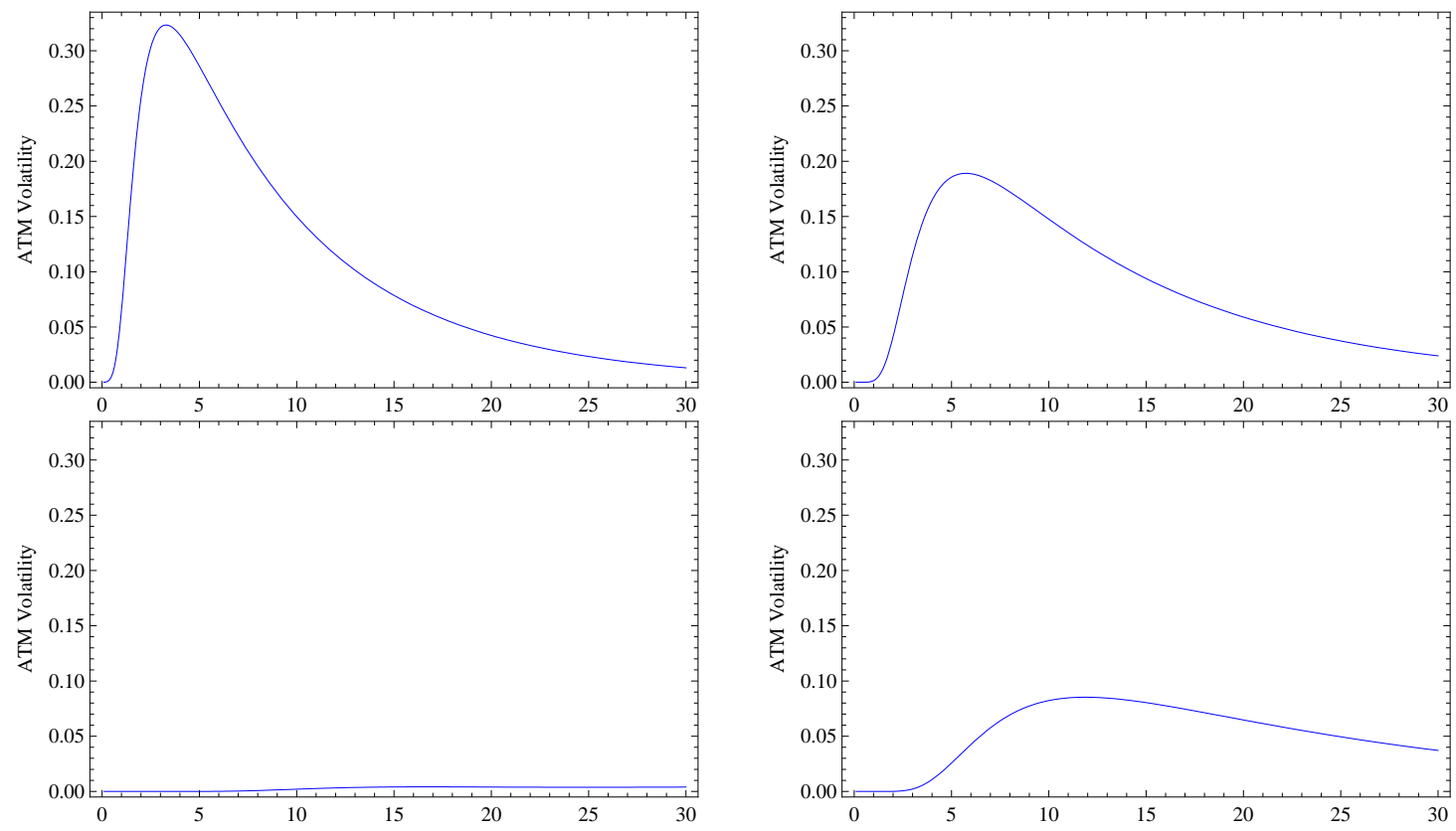

Figure 7: ATM interest rate caplet implied volatility term structure with $\bar{T} \in$ $[0,30]$ for $\beta \in\{0.0,0.25,0.5,0.75\}$, clockwise.

putative risk-neutral prices are not usually the same under the given modified constant elasticity of variance (MCEV) model. This is because the Radon-Nikodym derivative of the putative risk-neutral measure is a strict supermartingale. $\mathrm{Nu}-$ merical evaluation of the results reveal that the benchmark approach yields significantly lower real-world prices than corresponding putative risk-neutral prices of medium- and long-term contingent claims.

\section{Appendix A}

For a central chi-square random variable with $\nu \geq 0$ degrees of freedom, the probability density is $p_{\chi^{2}}(u ; \nu)$ and its distribution function $\chi^{2}(u ; \nu)$, as defined in Johnson, Kotz \& Balakrishnan (1994). We also introduce the gamma function $\Gamma(\alpha)$, the incomplete gamma function $\Gamma(u ; \alpha)$ and the regularised incomplete gamma function $\mathcal{Q}(u ; \alpha)$ for $u \geq 0$ and $\alpha>-1$, as given in Abramowitz \& Stegun (1970). The inverse of the regularised incomplete gamma function $\mathcal{Q}^{-1}(\tilde{p} ; \alpha)$ is such that $\tilde{p}=\mathcal{Q}(u ; \alpha)$.

Johnson, Kotz \& Balakrishnan (1995) define a non-central chi-square distributed random variable with $\nu$ degrees of freedom and non-centrality parameter $\lambda$ with the probability density

$$
p_{\chi^{2}}(x ; \nu, \lambda)=\sum_{\ell=0}^{\infty} \frac{\exp \left\{-\frac{\lambda}{2}\right\}\left(\frac{\lambda}{2}\right)^{\ell}}{\ell !} \frac{\left(\frac{x}{2}\right)^{\frac{\nu}{2}+\ell-1} \exp \left\{-\frac{x}{2}\right\}}{2 \Gamma\left(\frac{\nu}{2}+\ell\right)}
$$


for $x>0, u \geq 0, \nu \geq 0$ and $\lambda \geq 0$. The corresponding distribution function is given by $\chi^{2}(u ; \nu, \lambda)$. The density (A.1) can be rewritten as

$$
p_{\chi^{2}}(x ; \nu, \lambda)=\frac{1}{2}\left(\frac{x}{\lambda}\right)^{\frac{\nu-2}{4}} \exp \left\{-\frac{\lambda+x}{2}\right\} I_{\frac{\nu-2}{2}}(\sqrt{\lambda x})
$$

for $x \geq 0, \nu \geq 0$ and $\lambda \geq 0$, using the modified Bessel function of the first kind

$$
I_{d}(w)=\left(\frac{w}{2}\right)^{d} \sum_{\ell=0}^{\infty} \frac{\left(\frac{w}{2}\right)^{2 \ell}}{\ell ! \Gamma(\ell+d+1)} .
$$

A comparison of the transition density associated with the MCEV model in (3.14) with (A.2) reveals that

$$
\begin{aligned}
& \mathfrak{L}_{\bar{T}}^{i}=\frac{z_{t}^{i}}{\left(\tau_{\bar{T}}^{i}-\tau_{t}^{i}\right) A_{t}^{i}}=\frac{Z_{t}^{i}}{\left(\tau_{\bar{T}}^{i}-\tau_{t}^{i}\right) A_{t}^{i}}=\frac{\left(S_{t}^{i, \delta_{*}}\right)^{2\left(1-\beta_{i}\right)}}{\left(\tau_{\bar{T}}^{i}-\tau_{t}^{i}\right) A_{t}^{i}} \\
& \mathfrak{u}_{\bar{T}}^{i}=\frac{z_{\bar{T}}^{i}}{\left(\tau_{\bar{T}}^{i}-\tau_{t}^{i}\right) A_{\bar{T}}^{i}}=\frac{Z_{\bar{T}}^{i}}{\left(\tau_{\bar{T}}^{i}-\tau_{t}^{i}\right) A_{\bar{T}}^{i}}=\frac{\left(S_{\bar{T}}^{i, \delta_{*}}\right)^{2\left(1-\beta_{i}\right)}}{\left(\tau_{\bar{T}}^{i}-\tau_{t}^{i}\right) A_{\bar{T}}^{i}}
\end{aligned}
$$

will recover the two equations.

Central chi-square random variables are a specific case of the more general gamma distributed random variables. The probability density $p_{\mathcal{G}}\left(\frac{x}{\beta} ; \alpha\right)$ is defined as

$$
p_{\mathcal{G}}\left(\frac{x}{\beta} ; \alpha\right)=\frac{\exp \left\{-\frac{x}{\beta}\right\}\left(\frac{x}{\beta}\right)^{\alpha-1}}{\beta \Gamma(\alpha)}
$$

for $x \geq 0, \alpha>0$ and $\beta>0$. We also introduce the complementary distribution function $\mathcal{G}\left(\frac{u}{\beta} ; \alpha\right)$, defined as

$$
\mathcal{G}\left(\frac{u}{\beta} ; \alpha\right)=\int_{u}^{\infty} p_{\mathcal{G}}\left(\frac{x}{\beta} ; \alpha\right) d x
$$

for $u \geq 0, \alpha>0$ and $\beta>0$.

\section{Appendix B}

Proof of Lemma 4.1: Set $z_{t}^{i}=Z_{t}^{i}$ and $z_{\bar{T}}^{i}=Z_{\bar{T}}^{i}$ in (3.4), then from (4.1) we obtain

$$
P^{i}(t, \bar{T})=\int_{0}^{\infty}\left(\frac{z_{t}^{i}}{z_{\bar{T}}^{i}}\right)^{\frac{\mathfrak{n}_{i}-2}{2}} \mathfrak{q}_{\mathfrak{n}_{i}}\left(t, z_{t}^{i} ; \bar{T}, z_{\bar{T}}^{i}\right) d z_{\bar{T}}^{i}
$$

for $t \in[0, \bar{T}]$ and $i \in\{0,1, \ldots, d\}$. Use (A.4)-(A.5), (3.10) and algebra to obtain (4.5). 
Proof of Lemma 5.1: Apply the real-world pricing formula (2.7) to a call option payoff on the GOP, thereby obtaining

$$
\begin{aligned}
c_{\bar{T}, K, S^{i, \delta_{*}}}^{i}(t) & =E\left[\frac{S_{t}^{i, \delta_{*}}}{S_{\bar{T}}^{i, \delta_{*}}}\left(S_{\bar{T}}^{i, \delta_{*}}-K\right)^{+} \mid \mathcal{A}_{t}\right] \\
& =S_{t}^{i, \delta_{*}} E\left[\mathbf{1}\left\{S_{\bar{T}}^{i, \delta_{*}} \geq K\right\} \mid \mathcal{A}_{t}\right]-K E\left[\frac{S_{t}^{i, \delta_{*}}}{S_{\bar{T}}^{i, \delta_{*}}} \mathbf{1}\left\{S_{\bar{T}}^{i, \delta_{*}} \geq K\right\} \mid \mathcal{A}_{t}\right] \\
& =A_{\bar{T}, K, S^{i, \delta_{*}}}^{i,+}(t)-K B_{\bar{T}, K, S^{i, \delta_{*}}}^{i,+}(t)
\end{aligned}
$$

for $t \in[0, \bar{T}]$ and $i \in\{0,1, \ldots, d\}$. Set $z_{t}^{i}=Z_{t}^{i}$ and $z_{\bar{T}}^{i}=Z_{\bar{T}}^{i}$, use (3.4), (3.10) and (3.14) to determine an asset binary call option on the GOP as

$$
A_{\bar{T}, K, S^{i, \delta_{*}}}^{i,+}(t)=S_{t}^{i, \delta_{*}} \int_{K^{\frac{2}{\mathfrak{n}_{i}-2}}}^{\infty} \mathfrak{q}_{\mathfrak{n}_{i}}\left(t, z_{t}^{i} ; \bar{T}, z_{\bar{T}}^{i}\right) d z_{\bar{T}}^{i}
$$

whilst the corresponding bond binary call option on the GOP is

$$
B_{\bar{T}, K, S^{i, \delta_{*}}}^{i,+}(t)=\int_{K^{\frac{2}{\mathfrak{n}_{i}-2}}}^{\infty}\left(\frac{z_{t}^{i}}{z_{\bar{T}}^{i}}\right)^{\frac{\mathfrak{n}_{i}-2}{2}} \mathfrak{q}_{\mathfrak{n}_{i}}\left(t, z_{t}^{i} ; \bar{T}, z_{\bar{T}}^{i}\right) d z_{\bar{T}}^{i}
$$

for $t \in[0, \bar{T}]$ and $i \in\{0,1, \ldots, d\}$. The corresponding put option price can be found using the put-call parity relationship (5.5).

Proof of Lemma 6.1: Apply the real-world pricing formula (2.7) to a put option payoff on an exchange price to yield

$$
\begin{aligned}
p_{\bar{T}, K, X^{i, j}}^{i}(t) & =E\left[\frac{S_{t}^{i, \delta_{*}}}{S_{\bar{T}}^{i, \delta_{*}}}\left(K-X_{\bar{T}}^{i, j}\right)^{+} \mid \mathcal{A}_{t}\right] \\
& =-X_{t}^{i, j} E\left[\frac{S_{t}^{j, \delta_{*}}}{S_{\bar{T}}^{j, \delta_{*}}} \mathbf{1}\left\{X_{\bar{T}}^{i, j}<K\right\} \mid \mathcal{A}_{t}\right]+K E\left[\frac{S_{t}^{i, \delta_{*}}}{S_{\bar{T}}^{i, \delta_{*}}} \mathbf{1}\left\{X_{\bar{T}}^{i, j}<K\right\} \mid \mathcal{A}_{t}\right] \\
& =-A_{\bar{T}, K, X^{i, j}}^{i,-}(t)+K B_{\bar{T}, K, X^{i, j}}^{i,-}(t)
\end{aligned}
$$

for $t \in[0, \bar{T}]$ and $i, j \in\{0,1, \ldots, d\}$.

First, assume that the BESQ processes underlying each GOP denomination have identical dimensions, that is $\mathfrak{n}=\mathfrak{n}_{i}=\mathfrak{n}_{j}$, which obviously implies $\beta=\beta_{i}=\beta_{j}$ for all $i, j \in\{0,1, \ldots, d\}$. Second, assume that different currency denominations of the GOP are independent. Third, substitute $z_{t}^{k}=Z_{t}^{k}$ and $z_{\bar{T}}^{k}=Z_{\bar{T}}^{k}$ for $k \in\{i, j\}$ to obtain the asset binary put option relation

$$
A_{\bar{T}, K, X^{i, j}}^{i,-}(t)=X_{t}^{i, j} \int_{0}^{\infty} \int_{\frac{z_{\bar{T}}^{i}}{K^{\frac{2}{\mathfrak{n}-2}}}}^{\infty}\left(\frac{z_{t}^{j}}{z_{\bar{T}}^{j}}\right)^{\frac{\mathfrak{n}-2}{2}} \mathfrak{q}_{\mathfrak{n}}\left(t, z_{t}^{j} ; \bar{T}, z_{\bar{T}}^{j}\right) \mathfrak{q}_{\mathfrak{n}}\left(t, z_{t}^{i} ; \bar{T}, z_{\bar{T}}^{i}\right) d z_{\bar{T}}^{j} d z_{\bar{T}}^{i}
$$


and the corresponding bond binary put option relation of

$$
B_{\bar{T}, K, X^{i, j}}^{i,-}(t)=\int_{0}^{\infty} \int_{z_{\bar{T}}^{j} K^{\frac{2}{\mathfrak{n}-2}}}^{\infty}\left(\frac{z_{t}^{i}}{z_{\bar{T}}^{i}}\right)^{\frac{\mathfrak{n}-2}{2}} \mathfrak{q}_{\mathfrak{n}}\left(t, z_{t}^{i} ; \bar{T}, z_{\bar{T}}^{i}\right) \mathfrak{q}_{\mathfrak{n}}\left(t, z_{t}^{j} ; \bar{T}, z_{\bar{T}}^{j}\right) d z_{\bar{T}}^{i} d z_{\bar{T}}^{j}
$$

for $t \in[0, \bar{T}]$ and $i, j \in\{0,1, \ldots, d\}$. Now we use the substitutions (A.4)-(A.5) in both currency denominations together with the symmetry property of the modified Bessel function of the first kind, $I_{\mathfrak{d}}(\cdot)=I_{-\mathfrak{d}}(\cdot)$ for all $\mathfrak{d} \in\{\ldots,-1,0,+1, \ldots\}$, and some algebra to yield (6.2). The corresponding call option price is found via the put-call parity relationship (6.4).

Proof of Lemma 7.1: Apply the real-world pricing formula (2.7) to a call option payoff on a forward zero-coupon bond $P^{i}(\bar{T}, T)$ to obtain

$$
\begin{aligned}
c_{\bar{T}, K, P^{i}(\bar{T}, T)}^{i}(t) & =E\left[\frac{S_{t}^{i, \delta_{*}}}{S_{\bar{T}}^{i, \delta_{*}}}\left(P^{i}(\bar{T}, T)-K\right)^{+} \mid \mathcal{A}_{t}\right] \\
& =A_{\bar{T}, K, P^{i}(\bar{T}, T)}^{i,+}(t)-K B_{\bar{T}, K, P^{i}(\bar{T}, T)}^{i,+}(t)
\end{aligned}
$$

for $0 \leq t \leq \bar{T} \leq T$ and $i \in\{0,1, \ldots, d\}$. In the case of a bond binary call option we obtain

$$
B_{\bar{T}, K, P^{i}(\bar{T}, T)}^{i,+}(t)=E\left[\frac{S_{t}^{i, \delta_{*}}}{S_{\bar{T}}^{i, \delta_{*}}} \mathbf{1}_{\left\{P^{i}(\bar{T}, T) \geq K\right\}} \mid \mathcal{A}_{t}\right]=E\left[\frac{S_{t}^{i, \delta_{*}}}{S_{\bar{T}}^{i, \delta_{*}}} \mathbf{1}_{\left\{S_{\bar{T}}^{i, \delta_{*}} \geq \mathfrak{p}_{i}\right\}} \mid \mathcal{A}_{t}\right]
$$

where

$$
\mathfrak{p}_{i}=\left(2 A_{\bar{T}}^{i}\left(\tau_{T}^{i}-\tau_{\bar{T}}^{i}\right) \mathcal{Q}^{-1}\left[\frac{G_{T}^{i}(\bar{T})-K}{G_{T}^{i}(\bar{T})} ; \frac{\mathfrak{n}_{i}-2}{2}\right]\right)^{\frac{\mathfrak{n}_{i}-2}{2}}
$$

for $0 \leq t \leq \bar{T} \leq T$ and $i \in\{0,1, \ldots, d\}$. Since the short rate is constant, the solution to (B.9) comes from the result for a bond binary option on the GOP.

The asset binary call option on a forward zero-coupon bond $P^{i}(\bar{T}, T)$ is found as

$$
\begin{aligned}
A_{\bar{T}, K, P^{i}(\bar{T}, T)}^{i,+}(t) & =E\left[\frac{S_{t}^{i, \delta_{*}}}{S_{\bar{T}}^{i, \delta_{*}}} P^{i}(\bar{T}, T) \mathbf{1}_{\left\{P^{i}(\bar{T}, T) \geq K\right\}} \mid \mathcal{A}_{t}\right] \\
& =G_{T}^{i}(\bar{T}) E\left[\frac{B_{t}^{i}}{B_{\bar{T}}^{i}} \frac{\bar{S}_{t}^{i, \delta_{*}}}{\bar{S}_{\bar{T}}^{i, \delta_{*}}} M_{T}^{i}(\bar{T}) \mathbf{1}_{\left\{\bar{S}_{\bar{T}}^{i, \delta_{*}} \geq \bar{p}_{i}\right\}} \mid \mathcal{A}_{t}\right] \\
& =G_{T}^{i}(t) \chi^{2}\left(\mathfrak{L}_{\bar{T}}^{i} ; \mathfrak{n}_{i}-2, \tilde{\mathfrak{p}}_{i}\right)-\Xi_{\bar{T}, T, P^{i}(\bar{T}, T)}^{i,+}(t)
\end{aligned}
$$

for $0 \leq t \leq \bar{T} \leq T$ and $i \in\{0,1, \ldots, d\}$ using (4.7), (4.8) and (B.10) and notation (7.3). The special function $\Xi_{\bar{T}, T, P^{i}(\bar{T}, T)}^{i,+}(t)$ can be calculated using (3.4), (3.10) 
and substituting $z_{t}^{i}=Z_{t}^{i}$ and $z_{\bar{T}}^{i}=Z_{\bar{T}}^{i}$ as

$\Xi_{\bar{T}, T, P^{i}(\bar{T}, T)}^{i,+}(t)=\frac{B_{\bar{T}}^{i}}{B_{T}^{i}} \int_{\mathfrak{p}_{i}^{2\left(1-\beta_{i}\right)}}^{\infty}\left(\frac{z_{t}^{i}}{z_{\bar{T}}^{i}}\right)^{\frac{1}{2\left(1-\beta_{i}\right)}} \mathcal{Q}\left(\frac{z_{\bar{T}}^{i}}{2 A_{\bar{T}}^{i}\left(\tau_{T}^{i}-\tau_{\bar{T}}^{i}\right)} ; \frac{1}{1-\beta_{i}}\right) \mathfrak{q}_{\mathfrak{n}_{i}}\left(t, z_{t}^{i} ; \bar{T}, z_{\bar{T}}^{i}\right) d z_{\bar{T}}^{i}$

for $0 \leq t \leq \bar{T} \leq T$ and $i \in\{0,1, \ldots, d\}$.

For a put option on a forward zero-coupon bond $P^{i}(\bar{T}, T)$ it is advantageous to separate the solution into two cases because of restrictions imposed by parameter (7.4). For a bond binary put option on a zero-coupon bond, one obtains

$$
\begin{aligned}
B_{\bar{T}, K, P^{i}(\bar{T}, T)}^{i,-}(t) & =E\left[\frac{S_{t}^{i, \delta_{*}}}{S_{\bar{T}}^{i, \delta_{*}}} \mathbf{1}_{\left\{P^{i}(\bar{T}, T)<K\right\}} \mid \mathcal{A}_{t}\right] \\
& = \begin{cases}P^{i}(t, \bar{T})-B_{\bar{T}, K, P^{i}(\bar{T}, T)}^{i,+}(t) & \text { for } \quad K<G_{T}^{i}(\bar{T}) \\
P^{i}(t, \bar{T}) & \text { for } \quad K \geq G_{T}^{i}(\bar{T})\end{cases}
\end{aligned}
$$

for $0 \leq t \leq \bar{T} \leq T$ and $i \in\{0,1, \ldots, d\}$. The solution to (B.13) when $K<G_{T}^{i}(\bar{T})$ comes from (4.5) and (B.9), and when $K \geq G_{T}^{i}(\bar{T})$ the solution equals (4.5). The corresponding asset binary put option on a zero-coupon bond is found using iterated expectations, (4.5) and (B.11) to equal

$$
\begin{aligned}
A_{\bar{T}, K, P^{i}(\bar{T}, T)}^{i,-}(t) & =E\left[\frac{S_{t}^{i, \delta_{*}}}{S_{\bar{T}}^{i, \delta_{*}}} P^{i}(\bar{T}, T) \mathbf{1}_{\left\{P^{i}(\bar{T}, T) \leq K\right\}} \mid \mathcal{A}_{t}\right] \\
& = \begin{cases}P^{i}(t, T)-A_{\bar{T}, K, P^{i}(\bar{T}, T)}^{i,+}(t) & \text { for } \quad K<G_{T}^{i}(\bar{T}) \\
P^{i}(t, T) & \text { for } \quad K \geq G_{T}^{i}(\bar{T})\end{cases}
\end{aligned}
$$

for $0 \leq t \leq \bar{T} \leq T$ and $i \in\{0,1, \ldots, d\}$

\section{Acknowledgement}

The authors would like to thank Hardy Hulley, David Heath, and Leah Kelly for their interest in this research and constructive discussions on the subject.

\section{References}

Abramowitz, M. \& I. A. Stegun (Eds.) (1970). Handbook of Mathematical Functions (9th ed.), Volume 55 of Applied Mathematics Series. Washington DC: National Bureau of Standards.

Beckers, S. (1980). The constant elasticity of variance model and its implications for option pricing. J. Finance 35(3), 661-673. 
Black, F. (1976). Studies in stock price volatility changes. In Proceedings of the 1976 Business Meeting of the Business and Economic Statistics Section, American Statistical Association, pp. 177-181.

Boyle, P. P. \& Y. Tian (1999). Pricing lookback and barrier options under the CEV process. J. Financial and Quantitative Analysis 32(2), 241-264.

Brigo, D. \& F. Mercurio (2005). Interest Rate Models - Theory and Practice (2nd ed.). Springer Finance.

Bru, M.-F. (1991). Wishart processes. J. Theoret. Probab. 4(4), 725-751.

Cox, J. C. (1996). The constant elasticity of variance option pricing model. J. Portfolio Manag. (Special Issue), 15-17.

Cox, J. C. \& D. G. Hobson (2005). Local martingales, bubbles and option prices. Finance Stoch. 9, 477-492.

Cox, J. C., J. E. Ingersoll, \& S. A. Ross (1985). A theory of the term structure of interest rates. Econometrica 53, 385-407.

Cox, J. C. \& S. A. Ross (1976). The valuation of options for alternative stochastic processes. J. Financial Economics 3, 145-166.

Davydov, D. \& V. Linetsky (2001). Pricing and hedging path-dependent options under the CEV process. Management Science 47(7), 949-965.

Delbaen, F. \& W. Schachermayer (1994). A general version of the fundamental theorem of asset pricing. Math. Ann. 300, 463-520.

Delbaen, F. \& H. Shirakawa (2002). A note on option pricing for the constant elasticity of variance model. Asia-Pacific Financial Markets 9(2), 85-99.

Engel, D. C. \& J. D. MacBeth (1982). Further results on the constant elasticity of variance call option pricing model. J. Financial and Quantitative Analysis 17(4), 533-554.

Feller, W. (1951). Two singular diffusion problems. Ann. of Math. 54(1), 173182.

Heath, D. \& E. Platen (2002). Consistent pricing and hedging for a modified constant elasticity of variance model. Quant. Finance. 2(6), 459-467.

Heston, S. L., M. Loewenstein, \& G. A. Willard (2007). Options and bubbles. Rev. Financial Studies 20(2), 359-390.

Johnson, N. L., S. Kotz, \& N. Balakrishnan (1994). Continuous Univariate Distributions (2nd ed.), Volume 1 of Wiley Series in Probability and Mathematical Statistics. John Wiley \& Sons.

Johnson, N. L., S. Kotz, \& N. Balakrishnan (1995). Continuous Univariate Distributions (2nd ed.), Volume 2 of Wiley Series in Probability and Mathematical Statistics. John Wiley \& Sons.

Jones, C. S. (2003). The dynamics of stochastic volatility: Evidence from underlying and options markets. J. Econometrics 116, 181-224. 
Karatzas, I. \& S. E. Shreve (1998). Methods of Mathematical Finance, Volume 39 of Appl. Math. Springer.

Kelly, J. R. (1956). A new interpretation of information rate. Bell Syst. Techn. J. 35, 917-926.

Lewis, A. L. (2000). Option Valuation Under Stochastic Volatility. Finance Press, Newport Beach.

Linetsky, V. (2004). Lookback options and diffusion hitting times: A spectral expansion approach. Finance Stoch. 1, 373-398.

Lo, C. F., H. M. Tang, K. C. Ku, \& C. H. Hui (2004). Valuation CEV barrier options with time-dependent model parameters. In Proceedings of the 2nd IASTED International Conference on Financial Engineering and Applications. ACTA Press.

Lo, C. F., P. H. Yuen, \& C. H. Hui (2000). Constant elasticity of variance option pricing model with time dependent parameters. Int. J. Theor. Appl. Finance 3(4), 661-674.

Loewenstein, M. \& G. A. Willard (2000). Local martingales, arbitrage, and viability: Free snacks and cheap thrills. Econometric Theory 16(1), 135161.

Long, J. B. (1990). The numeraire portfolio. J. Financial Economics 26, 29-69.

MacBeth, J. D. \& L. J. Merville (1980). Tests of the Black-Scholes and Cox call option valuation models. J. Finance 35(2), 285-301.

Miller, S. \& E. Platen (2005). A two-factor model for low interest rate regimes. Asia-Pacific Financial Markets 11(1), 107-133.

Platen, E. (2002). Arbitrage in continuous complete markets. Adv. in Appl. Probab. 34(3), 540-558.

Platen, E. (2005). Diversified portfolios with jumps in a benchmark framework. Asia-Pacific Financial Markets 11(1), 1-22.

Platen, E. \& D. Heath (2006). A Benchmark Approach to Quantitative Finance. Springer Finance. Springer.

Schroder, M. (1989). Computing the constant elasticity of variance option pricing formula. J. Finance 44(1), 211-219.

Shaw, W. (1998). Pricing Derivatives with Mathematica. Cambridge University Press.

Sin, C. A. (1998). Complications with stochastic volatility models. Adv. in Appl. Probab. 30, 256-268.

Yor, M. (1992). On some exponential functionals of Brownian motion. Adv. in Appl. Probab. 24, 509-531. 